\title{
The innate immune receptor TREM-1 promotes liver injury and fibrosis
}

\author{
Anh Thu Nguyen-Lefebvre, ${ }^{1}$ Ashwin Ajith, ${ }^{1}$ Vera Portik-Dobos, ${ }^{1}$ Daniel David Horuzsko, ${ }^{1}$ Ali Syed Arbab, ${ }^{2}$ Amiran Dzutsev, ${ }^{3}$ \\ Ramses Sadek, ${ }^{4}$ Giorgio Trinchieri, ${ }^{3}$ and Anatolij Horuzsko'
}

${ }^{1}$ Molecular Oncology and Biomarkers Program, Georgia Cancer Center, Department of Medicine, Medical College of Georgia, Augusta University, Augusta, Georgia, USA. ${ }^{2}$ Tumor Angiogenesis Laboratory, Ceorgia Cancer Center, Department of Biochemistry and Molecular Biology, Augusta University, Augusta, Georgia, USA. ${ }^{3}$ Cancer and Inflammation Program, Center for Cancer Research, National Cancer Institute, NIH, Bethesda, Maryland, USA. ${ }^{4}$ Ceorgia Cancer Center, Augusta University, Augusta, Georgia, USA.

\begin{abstract}
Inflammation occurs in all tissues in response to injury or stress and is the key process underlying hepatic fibrogenesis. Targeting chronic and uncontrolled inflammation is one strategy to prevent liver injury and fibrosis progression. Here, we demonstrate that triggering receptor expressed on myeloid cells 1 (TREM-1), an amplifier of inflammation, promotes liver disease by intensifying hepatic inflammation and fibrosis. In the liver, TREM-1 expression was limited to liver macrophages and monocytes and was highly upregulated on Kupffer cells, circulating monocytes, and monocyte-derived macrophages in a mouse model of chronic liver injury and fibrosis induced by carbon tetrachloride $\left(\mathrm{CCl}_{4}\right)$ administration. TREM-1 signaling promoted proinflammatory cytokine production and mobilization of inflammatory cells to the site of injury. Deletion of Trem1 reduced liver injury, inflammatory cell infiltration, and fibrogenesis. Reconstitution of Trem1-deficient mice with Trem1-sufficient Kupffer cells restored the recruitment of inflammatory monocytes and the severity of liver injury. Markedly increased infiltration of liver fibrotic areas with TREM-1-positive Kupffer cells and monocytes/macrophages was found in patients with hepatic fibrosis. Our data support a role of TREM-1 in liver injury and hepatic fibrogenesis and suggest that TREM-1 is a master regulator of Kupffer cell activation, which escalates chronic liver inflammatory responses, activates hepatic stellate cells, and reveals a mechanism of promotion of liver fibrosis.
\end{abstract}

\section{Introduction}

Fibrosis is a highly evolved defense response resulting in encapsulation of an injury in adult tissue. Hepatic fibrosis occurs in association with chronic liver injury and hepatocellular death caused by, among others, viral hepatitis, alcohol abuse, and drugs or chemical insults (1-6).

The liver is a target of infectious pathogens that, via microbe-associated molecular pattern (MAMP) molecules, induce an inflammatory response (7). In acute liver infections, inflammatory cell infiltration and hepatocellular damage are observed (8, 9). Repetition and/or persistence of the injury induces an extensive chronic inflammatory reaction in the liver resulting in a scarring process that destroys the normal architecture of the organ, leading to fibrosis and cirrhosis (10). In addition to infectionattributable fibrosis, liver fibrosis also occurs in response to a wide range of stimuli that cause stress and injury leading to sterile chronic inflammation $(11,12)$.

Although inflammation is correlated with fibrosis progression $(1,13,14)$, the cellular and molecular mechanisms that promote liver injury and fibrogenesis remain enigmatic. The triggering receptor expressed on myeloid cells 1 (TREM-1) is involved in the control of acute and chronic inflammatory responses. TREM-1 is

Conflict of interest: The authors have declared that no conflicts of interest exist. License: Copyright 2018, American Society for Clinical Investigation.

Submitted: October 23, 2017; Accepted: August 21, 2018.

Reference information: J Clin Invest. 2018;128(11):4870-4883.

https://doi.org/10.1172/JCI98156. a cell-surface-activating receptor and a member of the Ig superfamily that potently amplifies inflammatory responses by inducing the secretion of inflammatory mediators (15). TREM-1 is expressed on neutrophils, monocytes, and macrophages, where it is upregulated during infection, cancer development, and following TLR engagement $(16,17)$. TREM-1 has a V-type extracellular domain, a charged transmembrane domain, and a short cytoplasmic tail. TREM-1 associates with the adaptor molecule DAP12, which is required for surface expression and signaling by TREM-1 (18). DAP12 mediates downstream signaling through a cytoplasmic ITAM domain, which recruits SYK and activates PI3K, phospholipase C, and Vav signaling $(16,19)$. Limited data are available on the role of TREM-1 in liver inflammation and fibrogenesis. TREM-1 is expressed in human tumor-activated hepatic stellate cells (HSCs), and its level of expression is a prognostic factor for poor survival in patients with hepatitis B-related hepatocellular carcinoma (HCC), an inflammation-associated malignancy (20).

Recently, we demonstrated that TREM-1 is instrumental for Kupffer cell activation in a diethylnitrosamine-induced model of liver carcinogenesis and that its deletion attenuates the development of HCC (21). Here, we provide the first evidence to our knowledge that TREM-1 signaling, by targeting a chronic inflammatory response and profibrogenic pathways, is essential for inducing inflammation-mediated liver injury and fibrogenesis. We determined that Kupffer cells play a fundamental role in chronic liver inflammation and fibrogenesis using a model for liver fibrosis induced by repetitive injections of carbon tetrachloride $\left(\mathrm{CCl}_{4}\right)$. This well-defined and -characterized model of chronic 
A
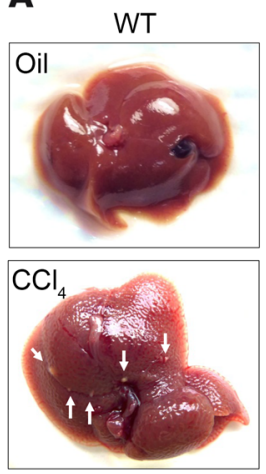

D
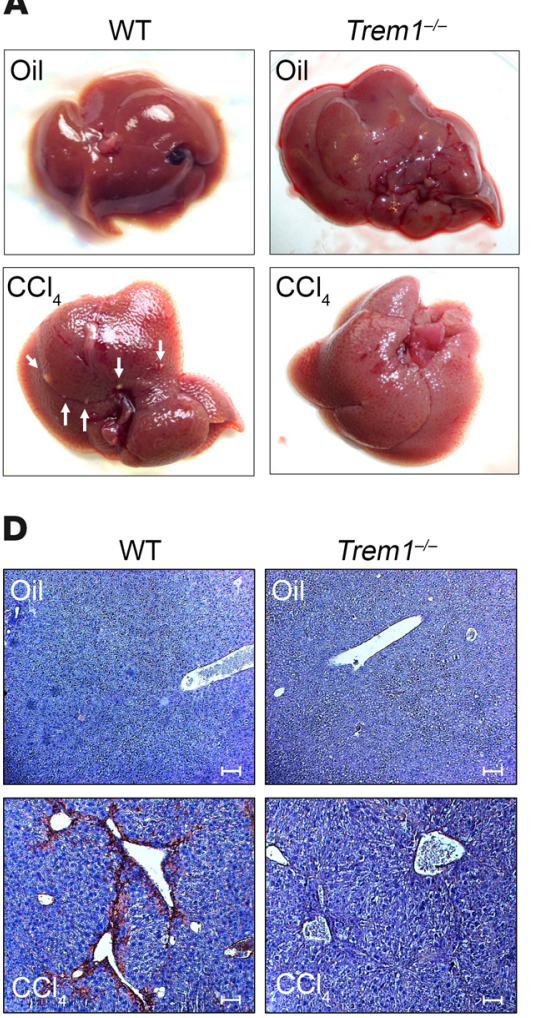

$\mathbf{F}$

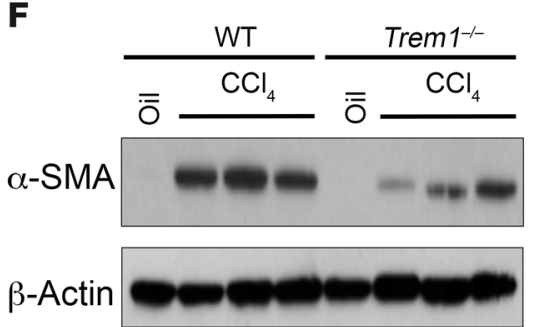

B

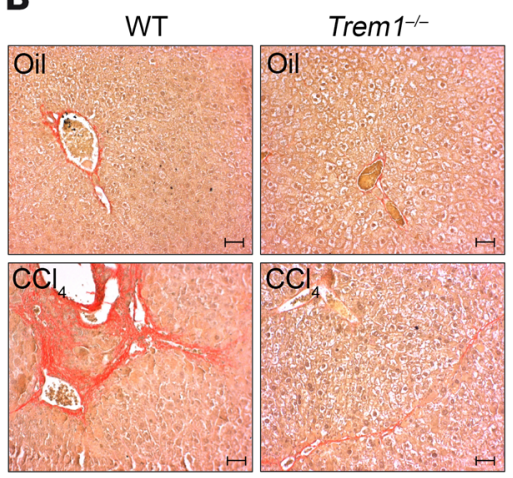

E

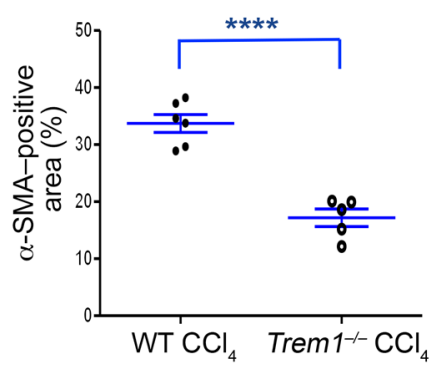

G

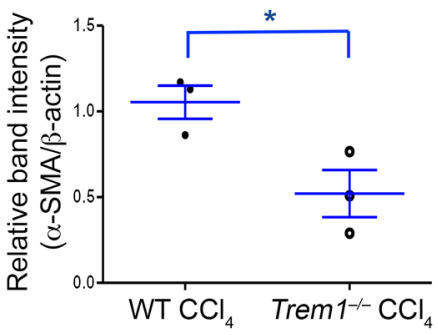

C

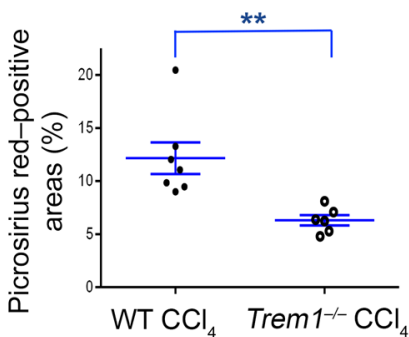

Figure 1. Deletion of Trem1 attenuates hepatic fibrogenesis. (A) Representative macroscopic images of livers from WT and Trem $^{-1 /-}$ control mice (oil-injected, $n=3$ /group, top) and WT and $\mathrm{Trem}^{1 /-}$ mice treated with 12 injections of $\mathrm{CCl}_{4}$ over a 6 -week period ( $n=6-7 /$ group, bottom). Arrowheads indicate fibrotic nodules visible on $\mathrm{CCl}_{4}$-treated WT mice. (B) Collagen deposition was evaluated with Picrosirius red staining. Representative images of liver sections from WT and Trem $1^{-1-}$ control mice ( $n=3$ /group, top) and from WT and Trem ${ }^{1 /-}$ mice treated with $\mathrm{CCl}_{4}$ ( $n=6-7 /$ group, bottom). Original magnification, $\times 20$; scale bars: $50 \mu \mathrm{m}$. (C) Quantification (percentage) of Picrosirius red-positive areas. (D) Representative images of liver sections from WT and Trem $1^{-/-}$control mice $(n=3$ / group, top) and from WT and Trem1/-- mice treated with $\mathrm{CCl}_{4}$ ( $n=5-6 /$ group, bottom) stained with anti- $\alpha$-SMA antibody. Original magnification, $\times 10$; scale bars: $100 \mu \mathrm{m}$. (E) Quantification of $\alpha$-SMA-positive areas (percentage). (F) Immunoblot analysis of $\alpha$-SMA in liver lysates from the indicated mice ( $n=3$ /group). $\beta$-Actin was used as a loading control. The full, uncut gels are shown in the supplemental material. (C) Quantification of $\alpha$-SMA expression ( $n=3$ mice/group). Results are displayed as the mean \pm SEM. ${ }^{*} P<0.05$, ${ }^{* *} P<$ 0.01 , and ${ }^{* * *} P<0.0001$, by 2-tailed Student's $t$ test (C, E, and $\mathbf{G}$ ). Experiments shown in $\mathbf{A}, \mathbf{B}$, and $\mathbf{D}$ are representative of 2 independent experiments. liver damage shares many features of chronic liver diseases in humans, including the development of liver fibrosis (22). TREM-1 has limited expression in healthy human liver. In contrast, in liver fibrotic areas from patients with advanced hepatic fibrosis, the number of TREM-1-positive liver macrophages and monocytes was markedly increased. In this study, we describe a TREM-1mediated mechanism of liver injury and fibrogenesis.

\section{Results}

TREM-1 enhances the development of liver fibrosis. A single dose of $\mathrm{CCl}_{4}$ induces centrilobular necrosis and reversible injury that triggers a wound-healing response (23). Repetitive administration of $\mathrm{CCl}_{4}$ promotes progressive fibrogenesis, cirrhosis, and, finally, HCC. To determine the role of TREM-1 in liver injury and fibrogenesis, Trem1 $1^{-/-}$and WT mice were injected with 12 doses of $\mathrm{CCl}_{4}$ over a 6-week period that in WT mice induced marked hepatic fibrosis. Trem1 $1^{-/}$mice showed significantly less fibrosis than did WT mice, as analyzed by (a) macroscopic appearance, (b) collagen deposition (Picrosirius red staining, $12.2 \% \pm 1.5 \%$ positive area for WT mice, $6.3 \% \pm 0.5 \%$ for Trem $^{-1-}$ mice), and (c) expression of $\alpha$-smooth muscle actin ( $\alpha$-SMA) (IHC, $33.7 \% \pm 1.6 \%$ for WT mice and $17.2 \% \pm 1.5 \%$ for Trem1 $1^{-/}$mice, Figure $\left.1, \mathrm{~A}-\mathrm{E}\right)$. A significant decrease of hepatic $\alpha$-SMA expression in Trem1 $1^{-1-}$ mice compared to WT mice was also observed by immunoblot analyses of total liver proteins (Figure 1, F and G). We also examined fibrosis development using bismuth oxide nanoparticles (Mvivo BIS), a contrast agent designed for small animal liver micro-CT imaging. Following administration, low doses of Mvivo BIS are rapidly taken up by the reticuloendothelial system in the liver, enabling high-definition imaging. After 6 weeks of $\mathrm{CCl}_{4}$ treatment, WT mice showed significantly less uptake of Mvivo BIS nanoparticles in the liver than did Trem1 $1^{-/-}$mice (Supplemental Figure 1, A and B; supplemental material available online with this article; https://oi.org/10.1172/JCI98156DS1). Alanine aminotransferase (ALT) and aspartate aminotransferase (AST) activities were measured as indicators of $\mathrm{CCl}_{4}$-induced liver injury. Both groups of mice showed elevated ALT and AST levels 6 weeks after $\mathrm{CCl}_{4}$ treatment. However, ALT and AST levels increased by more than 2-fold and 3-fold, respectively, in WT mice by week 6, while no significant increase was seen in $\mathrm{Trem}^{-/-}$mice, indicating a greater 
A
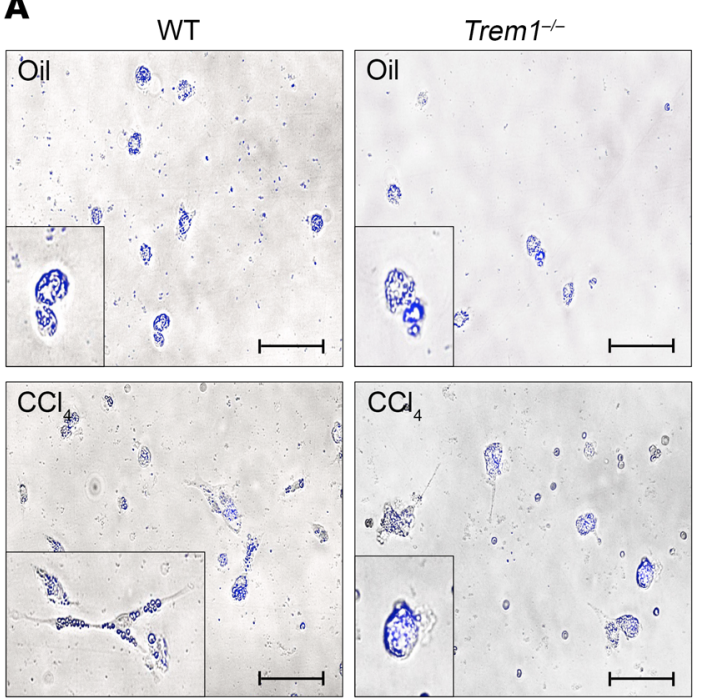

C
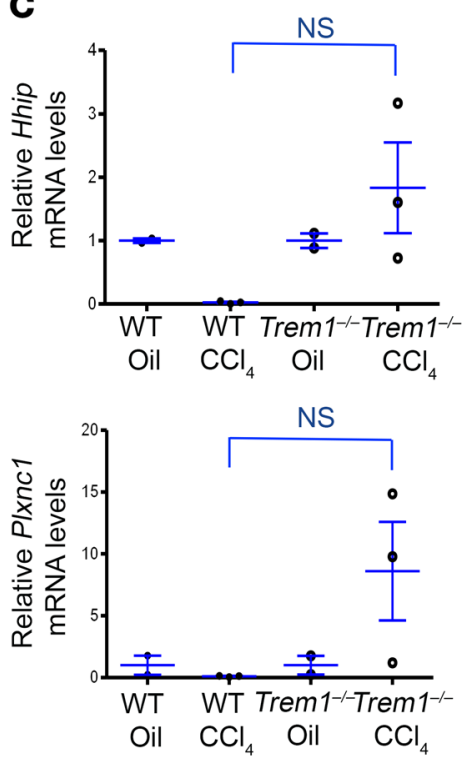

B
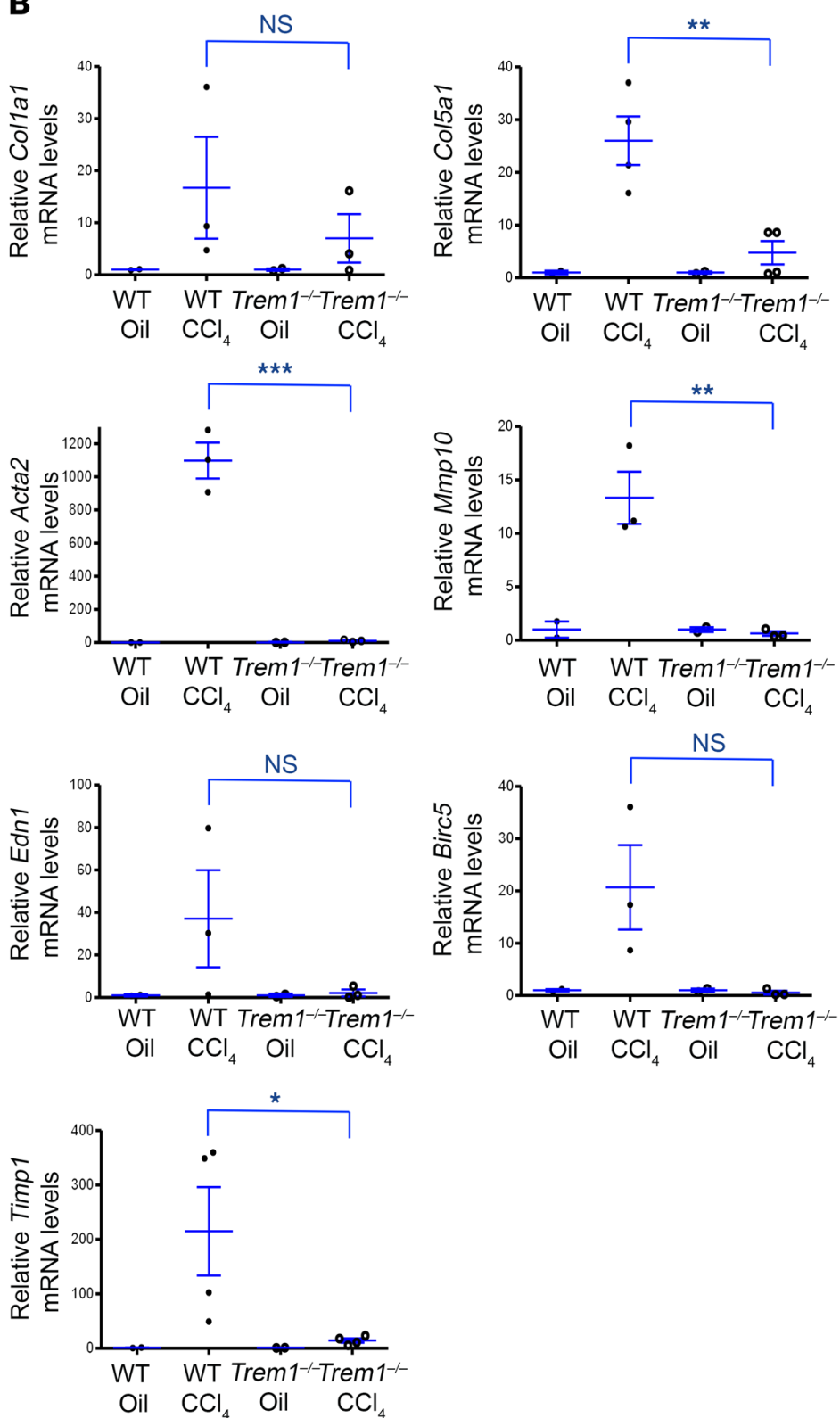$$
\text { (1) }
$$

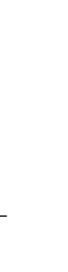

Figure 2. Deletion of Trem1 attenuates HSC activation and differentiation. HSCs were isolated from oil-injected (Oil) control WT and Trem $1^{-/-}$mice $(n=3 /$ group) and from WT and Trem1-- mice treated with $\mathrm{CCl}_{4}$ for 6 weeks ( $n=3-4 /$ group). (A) Representative images of freshly isolated HSCs from the indicated mice (oil-injected, top; $\mathrm{CCl}_{4}$-treated, bottom), visualized using a merging of phase-contrast microscopy and retinoid fluorescence (blue channel), show that HSCs from WT CCl-injured mice differentiated into myofibroblasts and lost their retinoic acid droplets. Original magnification, $\times 40 ;$ scale bars: $25 \mu \mathrm{m}$. Images shown are representative of 2 independent experiments. (B and C) Total RNA was isolated from HSC fractions from WT or Trem $1^{-1-}$ mice treated with CCl ${ }_{4}$ for 6 weeks ( $n=3-4$ /group). Col1a1, Col5a1, Acta2, Mmp10, Edn1, Birc5, Timp1, Hhip, and Plxnc1 mRNA levels were determined by RT-qPCR and are represented as the fold induction. Results are displayed as the mean \pm SEM. ${ }^{*} P<0.05,{ }^{* *} P<0.01$, and ${ }^{* * *} P<0.001$, by 2-tailed Student's $t$ test.

sensitivity to the development of liver injury in WT mice (Supplemental Figure 2). $\mathrm{CCl}_{4}$-treated WT mice showed significantly higher expression levels of fibrogenic genes that are upregulated in hepatic fibrosis than did Trem1 $1^{--}$mice, including $\alpha 1$ type 1 collagen (Colla1), $\alpha 2$ type 1 collagen (Col1a2), $\alpha 1$ type 3 collagen (Col3a1), $\alpha 1$ type 5 collagen (Col5a1), TGF- $\beta 1$ (Tgfb1), and $\alpha$-SMA (Acta2) (Supplemental Figure 3).

TREM-1 is essential for HSC activation. HSCs are the major collagen-producing cells in the fibrotic liver (1). Upon chronic liver injury, HSCs are activated to promote fibrogenesis by a wide range of signals from injured hepatocytes, activated Kupffer cells, inflammatory cells, and liver sinusoidal endothelial cells (LSECs). Upon activation, HSCs release vitamin A and lipid droplets and differentiate into myofibroblasts, which are elongated cells with fibrogenic and contractile activities (24, 25). In control (oiltreated) WT and Trem1 $1^{--}$mice, HSCs exhibit a quiescent phenotype and store vitamin A and lipid droplets, which display fading blue-green autofluorescence when excited with a light of approximately 405 to $407 \mathrm{~nm}$ and detected with a 450- to 50-nm bandpass filter (26) (Figure 2A, top). After 12 doses of $\mathrm{CCl}_{4}, \mathrm{HSCs}$ from 
A
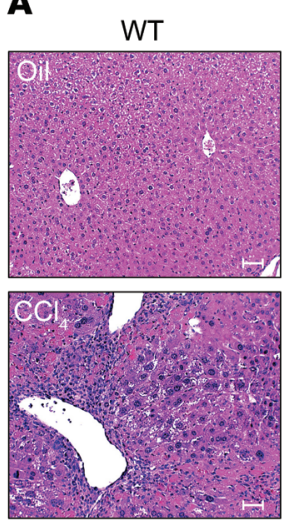

D
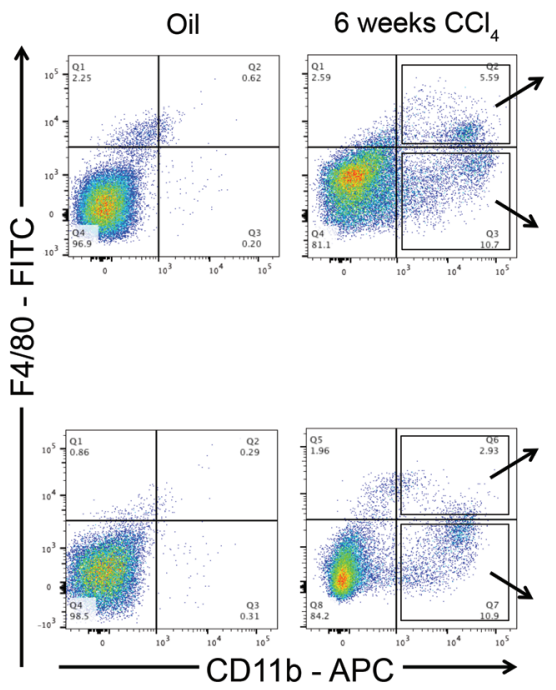

B
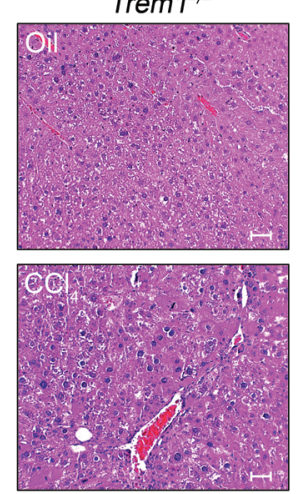

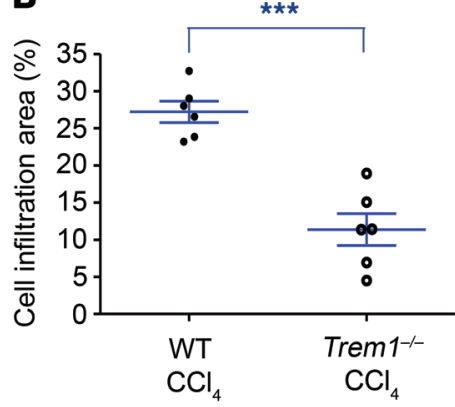

C
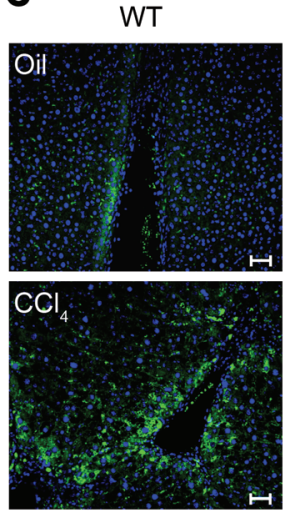

E
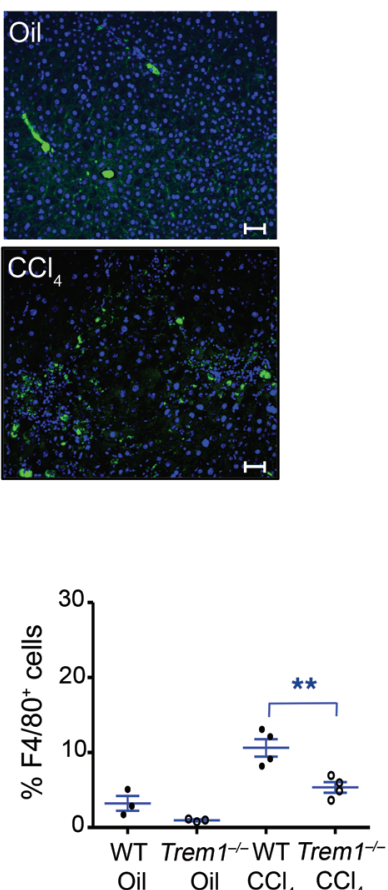
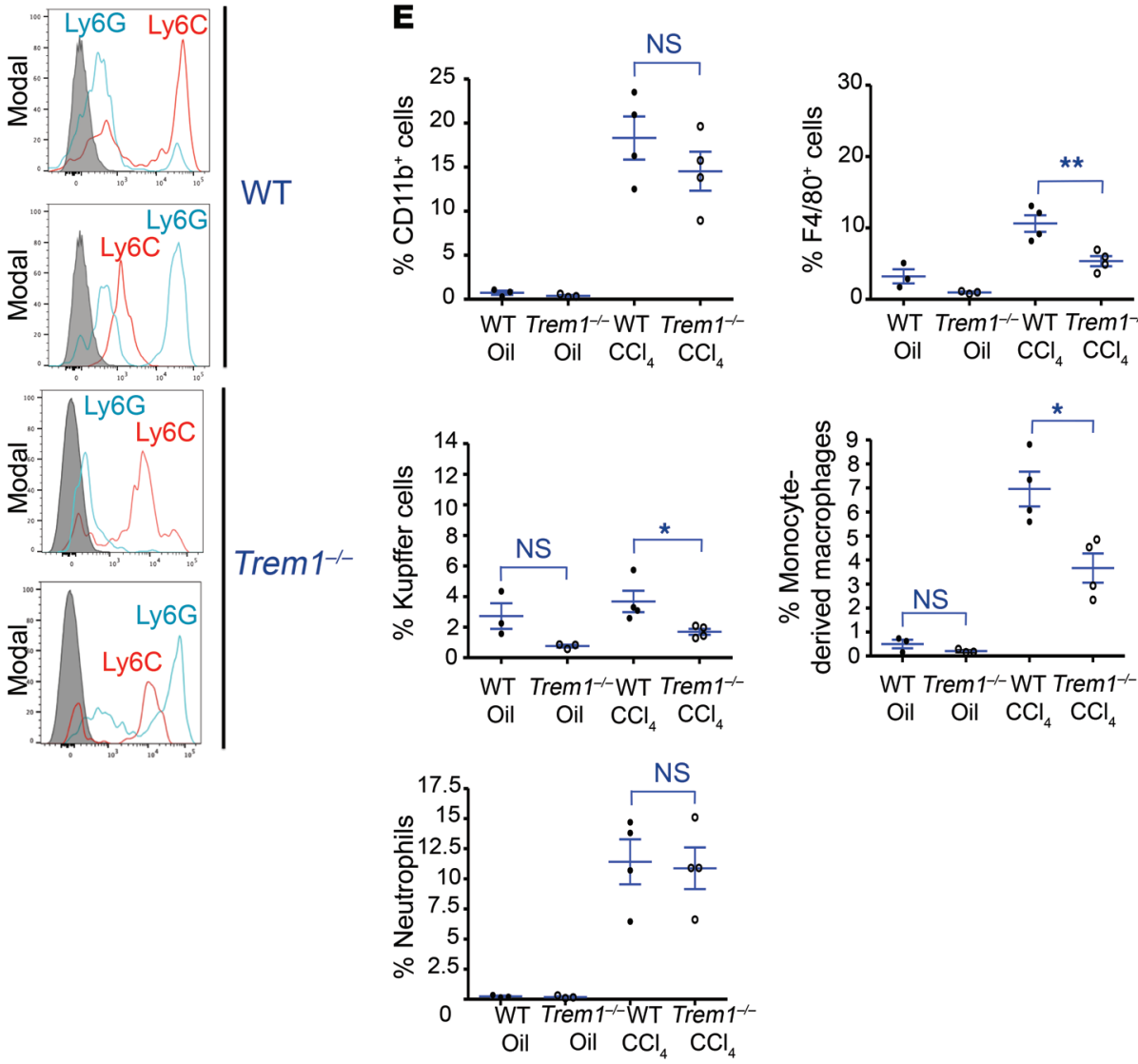

Figure 3. TREM-1 is essential for the recruitment and differentiation of monocyte-derived macrophages during hepatic fibrogenesis. WT and Trem $1^{-1-}$ mice were treated with 12 injections of $\mathrm{CCl}_{4}$ for 6 weeks. (A) Representative images of $\mathrm{H} \& \mathrm{E}$-stained liver sections stained from WT and Trem ${ }^{-/-}$control mice (oilinjected, $n=3$ /group, top) and WT and Trem $1^{-1-} \mathrm{CCl}_{4}$-injured mice ( $n=6 /$ group, bottom). Histology images revealed important mononuclear cell infiltration of fibrotic livers. Original magnification, $\times 20$; scale bars: $50 \mu \mathrm{m}$. (B) Quantification (percentage) of cell infiltration areas. (C) Representative images of FITCconjugated anti-F4/80 antibody-stained liver sections from control ( $n=3 /$ group, top) and $\mathrm{CCl}_{4}$-injured ( $n=6-7 /$ group, bottom) mice. Original magnification, $\times 20$; scale bars: $50 \mu \mathrm{m}$. (D) Flow cytometric dot plots of cells for identification of Kupffer cells (F4/80+CD11b-), monocyte-derived macrophages (F4/80+

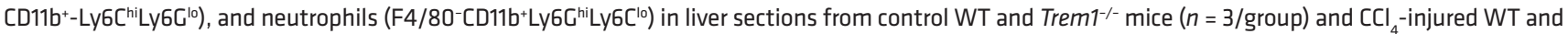
Trem1 ${ }^{-/-}$mice ( $n=4 /$ group). Control staining was performed with IgG isotype (gray histograms). APC, allophycocyanin. (E) Percentage of liver-infiltrated cell populations in WT and Trem $1^{1--}$ mice, analyzed by flow cytometry, after 6 weeks of oil or $\mathrm{CCl}_{4}$ treatment. Results are displayed as the mean $\pm \mathrm{SEM}$. ${ }^{*} P<0.05$, ${ }^{* *} P<0.01$, and ${ }^{* * *} P<0.001$, by 2-tailed Student's $t$ test (B and $\left.\mathbf{E}\right)$. Images in $\mathbf{A}$ and $\mathbf{C}$ are representative of 2 independent experiments.

WT mice showed characteristics of activated HSCs (Figure 2A, bottom left). In contrast, most HSCs from $\mathrm{CCl}_{4}$-treated Trem1 $1^{-1-}$ mice maintained a nonactivated vitamin A-rich round morphology (Figure 2A, bottom right). The role of TREM-1 in HSC activation was confirmed by the observation that mRNA expression of genes upregulated during cell activation and fibrogenesis
(Col5a1, Acta2, Mmp10, and Timp1) was significantly higher in $\mathrm{CCl}_{4}$-treated WT mice than in Trem1 ${ }^{-/}$mice (Figure 2B). Conversely, the transcript levels of genes that are downregulated in activated HSCs (Hhip encoding hedgehog-interacting protein and Plxnc1-encoding plexin C1) were lower in $\mathrm{CCl}_{4}$-treated WT mice than in Trem1 $1-$ mice (Figure 2C). 
A

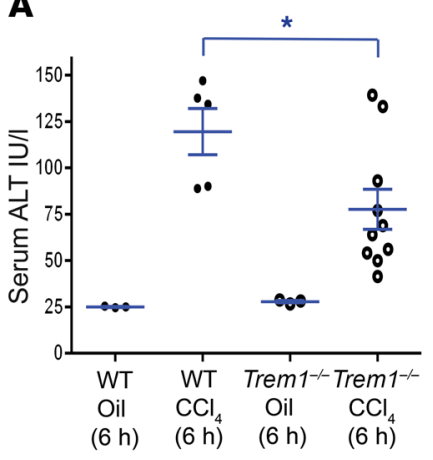

B
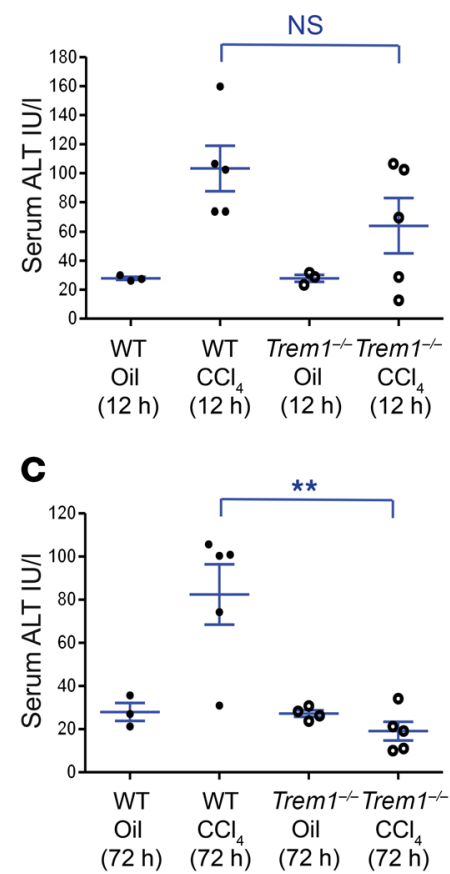

D

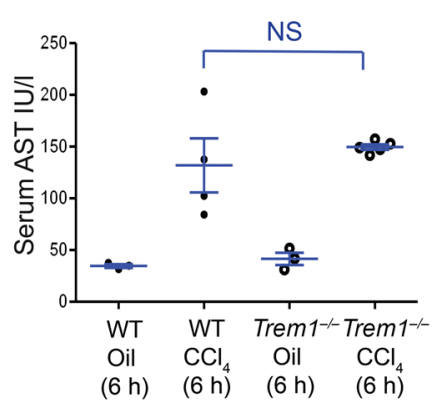

E

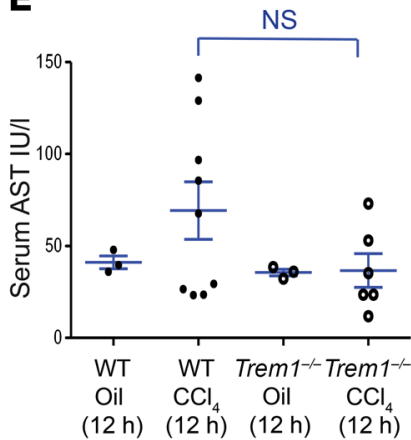

$\mathbf{F}$

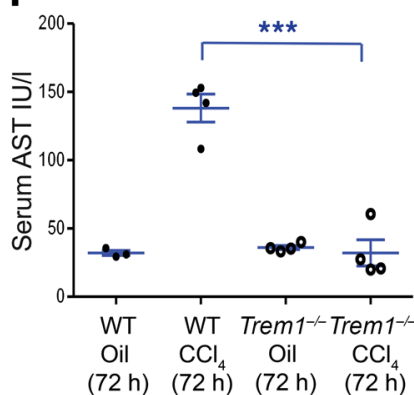

Figure 4. Deletion of Trem1 decreases liver injury at early stages of liver fibrogenesis. WT and Trem ${ }^{-/-}$mice were analyzed 6 hours, 12 hours, and 72 hours after injection of a single dose of $\mathrm{CCl}_{4}$. Serum ALT (A-C) and AST (D-F) levels for the indicated mice were measured with colorimetric assay ( $n=3-10$ mice/time point/group). Results are displayed as the mean \pm SEM. ${ }^{*} P<0.05,{ }^{* *} P<0.01$, and ${ }^{* *} P<0.001$, by 2 -tailed Student's $t$ test.

TREM-1 enhances hepatic inflammation during fibrogenesis. Treatment of WT mice with 12 doses of $\mathrm{CCl}_{4}$ elicited extensive changes in liver morphology, as observed in H\&E-stained tissue sections, including significantly increased cell infiltration surrounding islands of hepatocytes. In contrast, $\mathrm{CCl}_{4}$-treated Trem1 ${ }^{-/}$mice showed significantly reduced hepatic cell infiltration compared with WT mice (Figure 3, A and B). Fluorescence immunohistology showed, after $\mathrm{CCl}_{4}$ treatment, a substantially lower number of $\mathrm{F} 4 / 80$-positive cells in the livers of $\mathrm{Trem1}^{-/-}$mice than in WT mice (Figure 3C). Nonparenchymal cells isolated from WT and Trem1 $1^{-/}$livers were analyzed by flow cytometry to evaluate the $\mathrm{CCl}_{4}$-induced accumulation of myeloid cells that are required for HSC activation and hepatic fibrosis (27). While the number of cells expressing the pan-myeloid marker CD11b was similarly upregulated in both mouse strains by $\mathrm{CCl}_{4}$ treatment, the number

of $\mathrm{F} 4 / 80^{+}$cells was markedly reduced in $\mathrm{CCl}_{4}$-treated Trem1 ${ }^{--}$ mice compared with the number in WT mice (Figure 3, D and E). Liver-associated $\mathrm{F} 4 / 80^{+}$cells were composed of 2 populations: $\mathrm{F} 4 / 80^{+} \mathrm{CD} 11 \mathrm{~b}^{-}$cells, corresponding to resident Kupffer cells, and $\mathrm{F} 4 / 80^{+} \mathrm{CD} 11 \mathrm{~b}^{+}$cells. Analysis of the cell-surface markers Ly $6 \mathrm{C}$ and Ly $6 \mathrm{G}$ revealed that most $\mathrm{F} 4 / 80^{+} \mathrm{CD} 11 \mathrm{~b}^{+}$cells were inflammatory monocyte-derived macrophages $\left(\mathrm{F} 4 / 80^{+} \mathrm{CD} 11 \mathrm{~b}^{+} \mathrm{Ly} 6 \mathrm{C}^{\text {hi }} \mathrm{Ly} 6 \mathrm{G}^{\mathrm{lo}}\right)$ at different stages of differentiation (Figure 3D). The number of Kupffer cells and $\mathrm{F} 4 / 80^{+} \mathrm{CD} 11 \mathrm{~b}^{+}$cells was not significantly different in the livers of untreated WT and Trem1-- mice, but after $\mathrm{CCl}_{4}$ treatment, the numbers of both cell types increased and reached significantly higher levels in WT mice than in Trem1 $^{-1}$ mice (Figure $3 \mathrm{E}$ ). Most $\mathrm{F} 4 / 80^{-} \mathrm{CD} 11 \mathrm{~b}^{+}$cells expressed a high level of surface Ly6G (Ly6 $\mathrm{G}^{\mathrm{h}} \mathrm{Ly} 6 \mathrm{C}^{\mathrm{lo}}$ ) (Figure 3D) that identified them as neutrophils. These neutrophils were increased to a similar degree by $\mathrm{CCl}_{4}$ treatment in WT and Trem1 ${ }^{-/}$mice (Figure 3E). The significant increase in monocyte-derived macrophages $\left(\mathrm{F} 4 / 80^{+} \mathrm{CD} 11 \mathrm{~b}^{+}\right)$ in $\mathrm{CCl}_{4}$-treated WT mice compared with $\mathrm{Trem1}^{-1-}$ mice was confirmed in situ using a fluorescence multiplexed IHC assay (Supplemental Figure 4, A and B). In both groups of $\mathrm{CCl}_{4}$-treated mice, the abundance of adaptive immune cells such as $\mathrm{T}$ cells $\left(\mathrm{CD} 4^{+}, \mathrm{CD} 8^{+}\right)$ and B cells $\left(\mathrm{B}_{\left.22 \mathrm{O}^{+}\right)}\right.$was minimal (Supplemental Figure $\left.4 \mathrm{C}\right)$. These data demonstrate that during fibrosis development, TREM-1 signaling modulates myeloid hepatic inflammation, inducing an increased accumulation of monocyte-derived macrophages and resident Kupffer cells, but not affecting the number of neutrophils.

TREM-1 controls the mobilization of inflammatory cells in response to injury and consequently enhances liver damage. To analyze the role of TREM-1 in mediating the recruitment of inflammatory cells during fibrogenesis, we examined the early stage of $\mathrm{CCl}_{4}$-induced liver injury. $\mathrm{CCl}_{4}$ is quickly metabolized by liver cytochrome P450 enzymes into trichloromethyl free radicals that initiate a lipid peroxidation chain reaction leading to hepatocyte death and liver damage (28). Necrotic hepatocytes release damage-associated molecular pattern (DAMP) signaling molecules, including high-mobility group box 1 (HMGB1) protein and HSP70, that induce the activation, proliferation, and recruitment of inflammatory cells $(21,29-32)$, thus amplifying liver injury and establishing chronic inflammation. Both liver-resident cells and cells that are recruited in response to injury produce proinflammatory signals that contribute to the apoptotic and necrotic damage of hepatocytes. Injection of a single dose of $\mathrm{CCl}_{4}$ increases ALT and AST activity almost equivalently in WT and Trem1 $1^{-1-}$ mice at 6 hours and 12 hours (Figure 4, A, B, D, and E). However, while these increased levels were maintained in WT mice, they decreased and were reduced at 72 hours in $\mathrm{Treml}^{-1-}$ mice to the levels seen in untreated mice (Figure 4, C and F). These data indicate that TREM-1 signaling contributes to the persistence of the inflammatory response following $\mathrm{CCl}_{4}$ treatment and enhances liver injury.

The bone marrow is one of the sensors of liver injury. A single dose of $\mathrm{CCl}_{4}$ treatment induced only a modest perturbation of the number of total $\mathrm{CD} 11 \mathrm{~b}^{+}$cells, neutrophils, and monocytes in the bone marrow of Trem1 ${ }^{-/}$and WT mice (Supplemental Figure 5, A-D, and Supplemental Figure 6A). We analyzed peripheral blood cells 12 hours after $\mathrm{CCl}_{4}$ treatment and found a similar increase of CD11b-positive myeloid cells, including neutrophils and mono- 
A
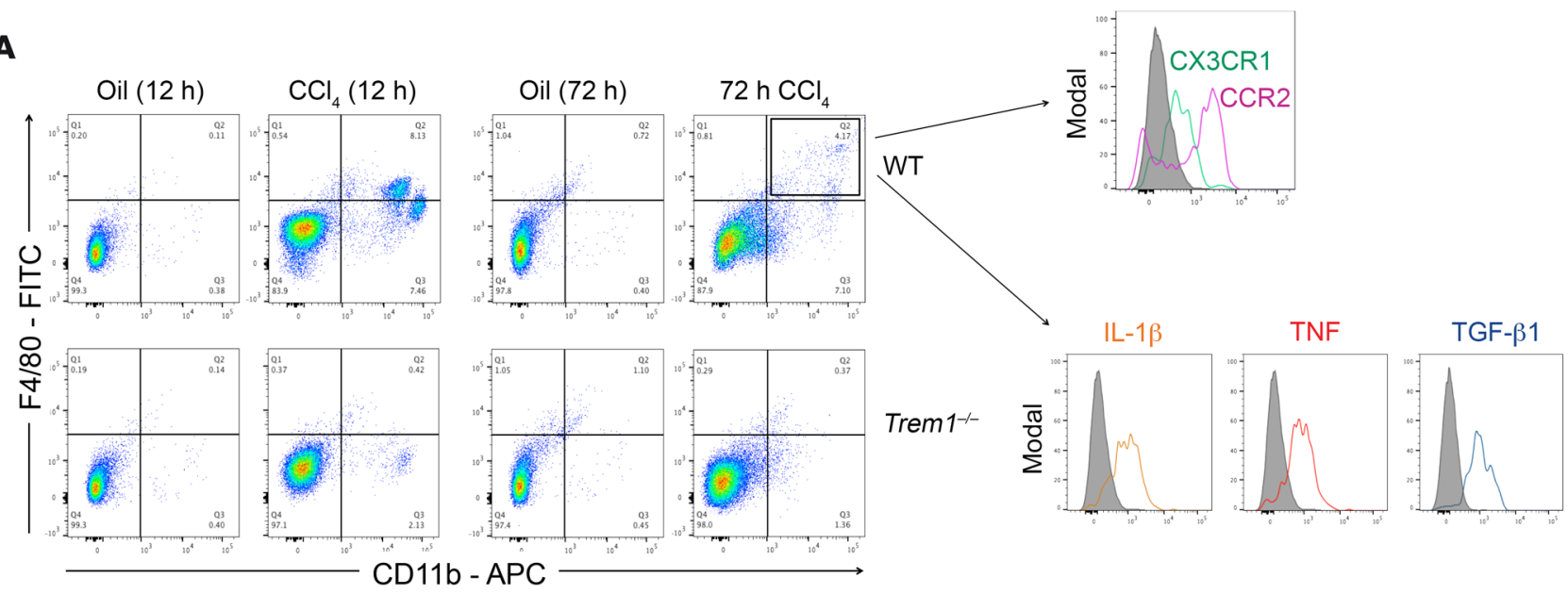

B

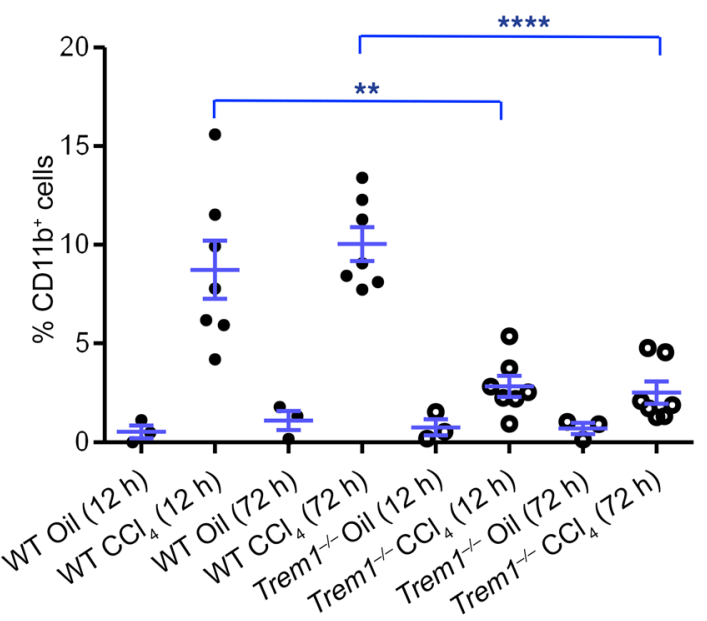

D

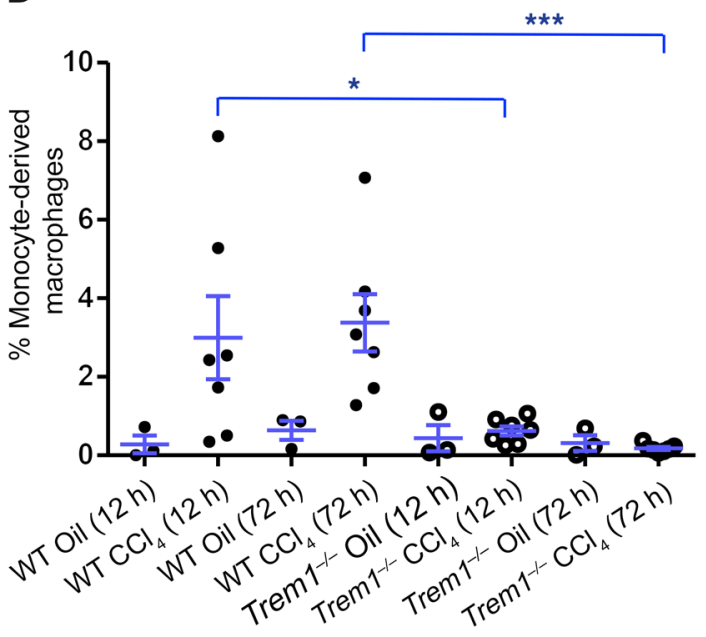

C

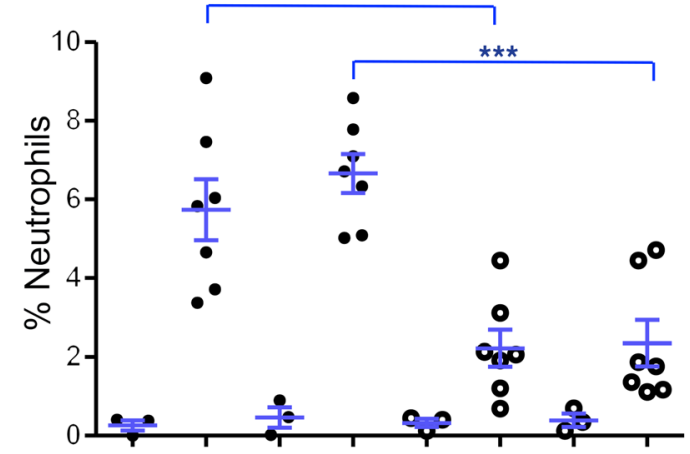

Figure 5. Deletion of Trem1 reduces inflammatory cell infiltration at early stages of liver fibrogenesis. (A) Flow cytometric dot plots of liver cells from control WT and Trem $^{1--}$ mice (oil-injected, $n=3 /$ group) and WT and Trem 1/- mice 12 hours and 72 hours after $\mathrm{CCl}_{4}$ injury ( $n=7$ mice/time point/group). Cells were stained with anti-F4/80, anti-CD11b, anti-CCR2, anti-CX3CR1, anti-IL-1 $\beta$, anti-TNF, and anti-TCF- $\beta 1$ antibodies. Liver cells from WT mice were stained and analyzed 72 hours after $\mathrm{CCl}_{4}$ injection for the expression of CCR2 and CX3CR1 ( $n=5$ mice) and for the intracellular expression of IL-1 $\beta$, TNF, and TGF- $\beta 1$ ( $n=7$ mice). Flow cytometric histograms represent cells gated on a $\mathrm{F} 4 / 80^{+} \mathrm{CD} 11 \mathrm{~b}^{+}$population. Control staining was performed with IgG isotype (gray histograms). (B-D) Percentage of CD11 $\mathrm{b}^{+}$cells, neutrophils, and inflammatory monocyte-derived macrophages in liver from WT and Trem1 $1^{-/}$mice quantified after 12 hours and 72 hours of oil injection ( $n=3$ mice/time point/group) or $\mathrm{CCl}_{4}$ treatment $(n=7 \mathrm{mice} /$ time point/ group). Results are displayed as the mean \pm SEM. ${ }^{*} P<0.05$, ${ }^{* *} P<0.01,{ }^{* * *} P<$ 0.001 , and ${ }^{* * *} P<0.0001$, by 2 -tailed Student's $t$ test (B, C, and $\left.\mathbf{D}\right)$.

cytes, in both groups of mice. However, 72 hours after $\mathrm{CCl}_{4}$ treatment, the frequency of myeloid cells in the blood was significantly higher in WT mice than in Trem1/-- mice (Supplemental Figure 5, $\mathrm{E}-\mathrm{H}$, and Supplemental Figure 6B). A much higher blood accumulation of patrolling monocytes $\left(\mathrm{CD} 11 \mathrm{~b}^{+} \mathrm{F} 4 / 80^{+} \mathrm{CCR} 2^{-} \mathrm{CX} 3 \mathrm{CR} 1^{+}\right)$ (33) was induced by $\mathrm{CCl}_{4}$ injection into WT mice compared with
Trem1 $1^{-/-}$mice (Supplemental Figure 5H). However, we observed the most dramatic changes in the livers of $\mathrm{CCl}_{4}$-treated mice. The number of myeloid cells, and especially neutrophils (Supplemental Figure 6C), progressively increased at both 12 hours and 72 hours and was significantly higher in livers from WT mice than in those from Trem1 $^{-/}$mice (Figure $5, \mathrm{~A}-\mathrm{C}$ ). The number of monocyte- 
A

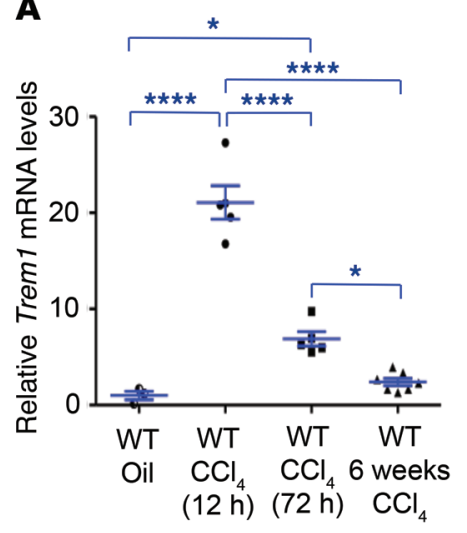

D
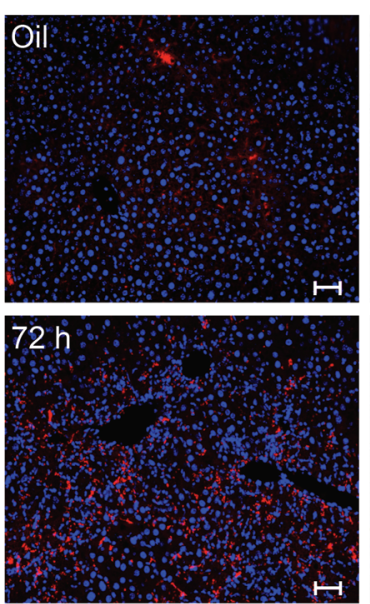

$\mathbf{F}$

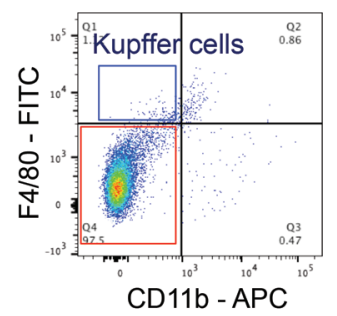

B

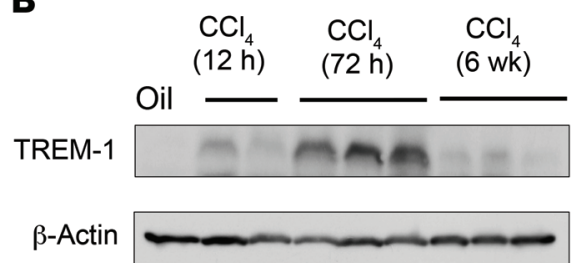

C
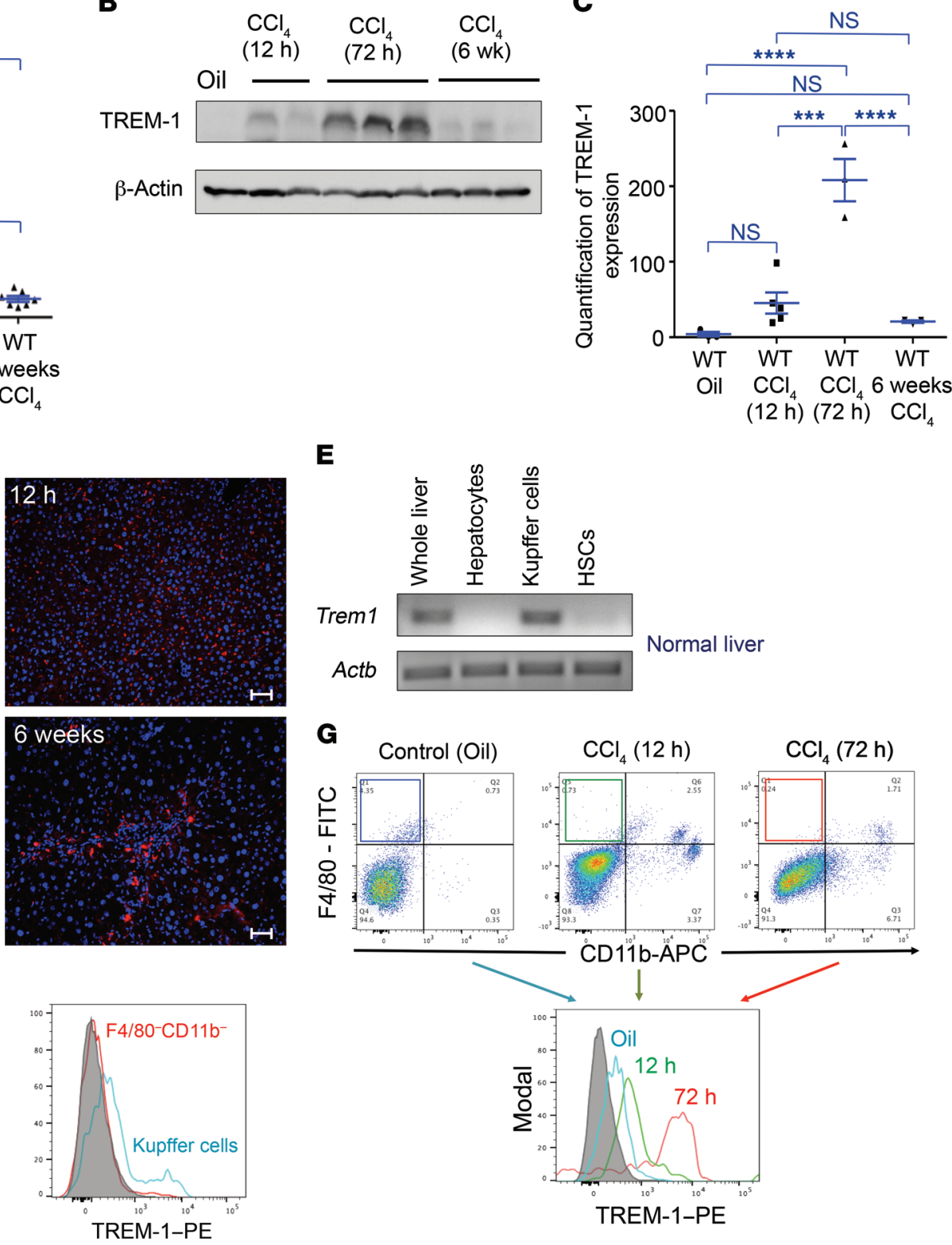

H

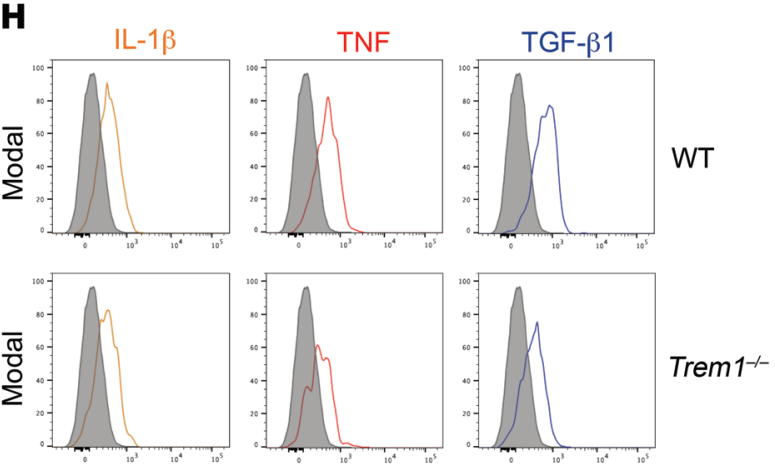

E
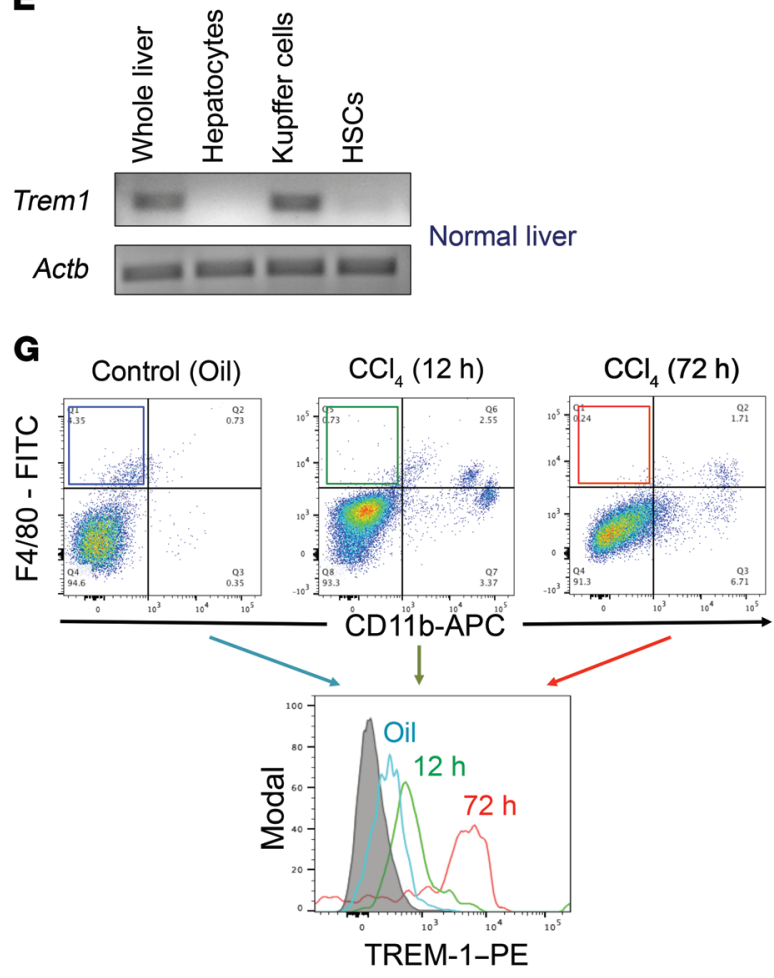

I

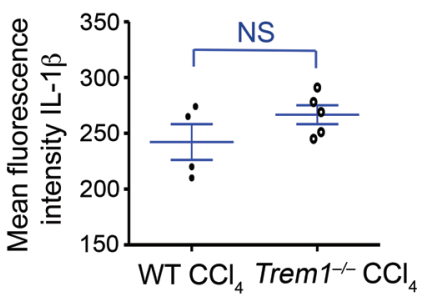

$(72 \mathrm{~h}) \quad$ (72 h)

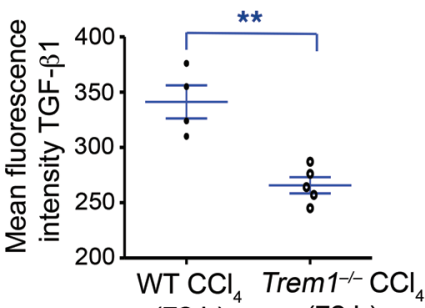

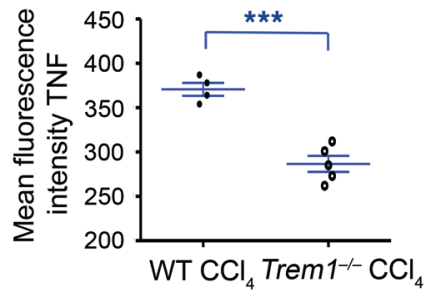

$(72 \mathrm{~h})$

(72 h) 
Figure 6. Expression of Trem1 in liver during fibrogenesis. (A) WT mice were treated with oil (control, $n=3$ ) or a single dose of $\mathrm{CCl}_{4}$ and analyzed after 12 hours and 72 hours ( $n=5$ mice/time point). Another group of WT mice was treated with 12 injections of $\mathrm{CCl}_{4}$ and analyzed after 6 weeks ( $n=7$ mice). mRNA levels of Trem1 in liver were assessed by RT-qPCR. Expression was normalized to the average of 3 different control genes (Actb, Gapdh, and Hprt1) and is represented as the fold induction. (B) Representative immunoblot analysis for TREM-1 in WT mouse liver lysates at different time points. $\beta$-Actin was used as a loading control. The full, uncut gels are shown in the supplemental material. (C) Quantification of TREM-1 expression in liver from WT control mice $(n=3)$ and $\mathrm{CCl}$-treated mice at 12 hours $(n=5), 72$ hours $(n=3)$, and 6 weeks $(n=3)$. (D) Representative phycoerythrin-conjugated (PE-conjugated) anti-TREM-1 antibody-stained immunofluorescence images of liver sections from WT control oil-injected mice ( $n=3$, top) and $\mathrm{CCl}_{4}$-treated mice at 12 hours (top), 72 hours, and 6 weeks ( $n=5-7$ mice/time point/ group, bottom). Original magnification, $\times 20$; scale bars: $50 \mu \mathrm{m}$. Images are representative of 2 independent experiments. (E) RT-qPCR was performed to assess Trem 1 mRNA expression in whole liver as well as in purified hepatocyte, Kupffer cell, and HSC fractions from WT mice ( $n=3$ mice/cell fraction). (F) Flow cytometric dot plots of WT control mouse liver cells ( $n=9$ mice) stained with anti-F4/80 and anti-CD11b antibodies. Flow cytometric histograms represent TREM- 1 expression on a gated F4/80-CD11 $b^{-}$cell population and on gated F4/80+CD11 $b^{-}$Kupffer cells ( $n=9$ mice). Control staining was performed with IgG isotype (gray histogram). (C) Flow cytometric dot plots and histograms of TREM-1 expression on gated $\mathrm{F} 4 / 80^{+} \mathrm{CD}_{11 \mathrm{~b}^{-}}$Kupffer cells isolated from oil-injected WT control mice $(n=9)$ and from mice treated with $\mathrm{CCl}_{4}(n=7$ mice/time point/group) for 12 hours and 72 hours. Control staining was performed with IgC isotype (gray histogram). (H) Flow cytometric histograms of intracellular expression of IL-1 $\beta$, TNF, and TCF- $\beta 1$ on gated F4/80+CD11b Kupffer cells from $\mathrm{CCl}_{4}$-injured $(72 \mathrm{~h}) \mathrm{WT}(n=4)$ and Trem1/-- $(n=5)$ mice. Control staining was performed with IgG isotype (gray histograms). (I) Quantification of mean fluorescence intensity of the indicated cytokines. Results are displayed as the mean \pm SEM. ${ }^{*} P<0.05$, ${ }^{* *} P<0.01$, ${ }^{* *} P<$ 0.001 , and ${ }^{* * *} P<0.0001$, by ANOVA followed by Bonferroni's post hoc test (A and $\mathbf{C}$ ) and 2-tailed Student's $t$ test (I).

derived macrophages $\left(\mathrm{CD} 11 \mathrm{~b}^{+} \mathrm{F} 4 / 80^{+} \mathrm{Ly}_{6} \mathrm{C}^{\text {hi }}\right)$ (Supplemental Figure $6 C$ ) was greatly increased in the injured livers of WT mice, but these monocyte-derived macrophages were almost absent in the livers of Trem1-- mice (Figure 5D). These cells were CCR2 $2^{+} \mathrm{CX} 3 \mathrm{CR} 1^{10}$ and produced IL-1 $\beta$, TNF, and TGF- $\beta 1$ (Figure $5 \mathrm{~A}$ ), which characterized them as infiltrating inflammatory monocyte-derived macrophages (34). The expression of genes involved in the recruitment and maintenance of inflammatory cells, including $C c l 2, C c l 7, C x c l 10$, Tnf, Illb, and $\mathrm{Il}$, was induced by $\mathrm{CCl}_{4}\left(12\right.$ hours and 72 hours after $\mathrm{CCl}_{4}$ injection) in the livers of WT mice, but not in those of Trem1 ${ }^{-/}$mice (Supplemental Figure 7). Thus, our data reveal a key role of TREM-1 in the mobilization, recruitment, and differentiation of inflammatory cells to the site of inflammation and injury.

TREM-1 expression in the liver and the profibrogenic signature of activated Kupffer cells increase during fibrogenesis. Total RNA was isolated from the livers of oil- or $\mathrm{CCl}_{4}$-injected WT mice and analyzed by real-time quantitative PCR (RT-qPCR). Trem 1 mRNA accumulation was increased at 12 hours, 72 hours, and 6 weeks after $\mathrm{CCl}_{4}$ treatment, with a maximum 20 -fold induction observed at 12 hours (Figure 6A). TREM-1 protein levels in the liver were also increased by $\mathrm{CCl}_{4}$ injection, with a peak detected at 72 hours (Figure 6, B and C). Fluorescence immunostaining of liver from WT mice at different time points confirmed the increase in TREM-1 expression in response to $\mathrm{CCl}_{4}$ (Figure 6D). To determine the cell types that express TREM-1 in normal liver, RT-PCR was performed on total RNA isolated from whole liver, purified hepatocytes, Kupffer cells, and HSCs from WT mice; we found that only Kupffer cells expressed Trem1 (Figure 6E). Flow cytometric analysis confirmed that TREM-1 protein was present only on Kupffer cells, and its density on these cells was increased at 12 hours, and to an even greater degree at 72 hours, in $\mathrm{CCl}_{4}$-induced liver injury (Figure 6, F and G). The increased levels of TREM-1 expression on Kupffer cells following $\mathrm{CCl}_{4}$ treatment may be responsible for the production by Kupffer cells of inflammatory chemokines, cytokines, and growth factors.

RNA-sequencing (RNA-seq) data showed that $\mathrm{CCl}_{4}$ treatment of WT mice resulted in a major shift of the transcriptomic profile of Kupffer cells, with approximately 1,000 genes differentially expressed, of which approximately 360 were increased and 640 decreased. Interestingly, the large majority of the genes that were induced or decreased by $\mathrm{CCl}_{4}$ treatment in WT mice were not modified or were modified to a significantly lesser degree than was seen in Trem1 $^{-1-}$ mice (Supplemental Figure 8A). The similarity between Kupffer cells from untreated WT mice and those from $\mathrm{CCl}_{4}$-treated $\mathrm{Trem1}^{-/}$mice was further demonstrated by correlation analysis of $\log _{2}$-transformed ratios of Kupffer cell transcripts from $\mathrm{CCl}_{4}$-treated WT mice versus those from $\mathrm{CCl}_{4}$-treated $\mathrm{Trem1}^{-/}$mice or untreated WT mice. The $R$ value between the 2 ratios was 0.76 , with a $P$ value of less than $10^{-4}$. Among the genes encoding growth factors that were upregulated by Kupffer cells in WT but not in Trem1 ${ }^{-/}$mice after $\mathrm{CCl}_{4}$ treatment were factors already shown to play a role in liver fibrosis, such as Tgfb1, Jag1 (jagged 1), and Osm (oncostatin M), as well as other factors, such as $\beta$-cellulin (Btc, a member of the EGF family), angiotensinogen (Agt), inhibin $\beta$ A (Inhba), and macrophage-stimulating 1 , hepatocyte growth factor-like (Mst1). The expression of genes encoding cytokines and chemokines was upregulated in WT mice, but not in Trem1 $1^{--}$mice. These included the cytokine gene Il23a and the chemokine genes $C c l 9, C x c l 2$, and $\mathrm{Cxcl} 3$, whose expressed proteins attract inflammatory and immune cell migration via CCR1 and CXCR2 and affect their processes of differentiation and function (Supplemental Figure $8 \mathrm{~B})$. In addition, total liver expression of TGF- $\beta 1$ was significantly higher in WT mice than in Trem1-/ mice (Supplemental Figure 9). These data support the notion that TGF- $\beta 1$ is the major regulator of liver fibrogenesis. We detected significantly decreased TNF and TGF- $\beta 1$ production in Kupffer cells from Trem1 ${ }^{--}$mice 72 hours after $\mathrm{CCl}_{4}$ injury, as shown in Figure 6, $\mathrm{H}$ and I. Lack of upregulation of the Ccl9, Cxcl2, Cxcl3, Illf9, and Il23a genes, as well as the decreased levels of TNF and TGF- $\beta 1$ in Trem1 $1^{--}$mice suggest that deletion of the Trem 1 gene alters Kupffer cell activation during the $\mathrm{CCl}_{4}$-induced inflammatory response.

Adoptive transfer of Trem1-sufficient Kupffer cells into Trem1deficient mice reconstitutes the impaired myeloid inflammatory response following $\mathrm{CCl}_{4}$-induced injury. To test whether the impaired activation of Kupffer cells in $\mathrm{Trem1}^{-1-}$ mice plays a major role in the reduced response of liver injury and recruitment of inflammatory cells to $\mathrm{CCl}_{4}$-induced liver damage, we depleted Kupffer cells in WT and Trem1 $1^{--}$mice by treatment with clodronate-containing liposomes (Figure 7A, top), followed by intravenous reconstitution with Kupffer cells isolated from WT mice and $\mathrm{CCl}_{4}$ injection (Figure 7A, bottom). We observed that a similar number of trans- 
A
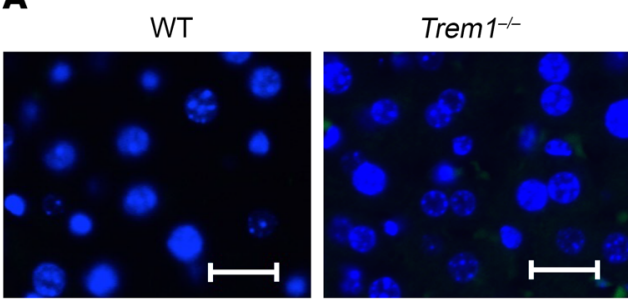

Kupffer cell depletion with clodronate-containing

$$
\text { liposomes }
$$
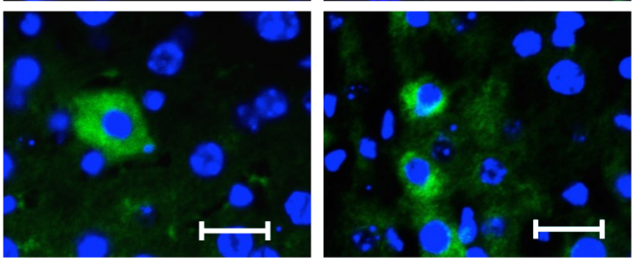

Kupffer cell depletion with clodronate-containing liposomes followed by transfer of WT Kupffer cells

D

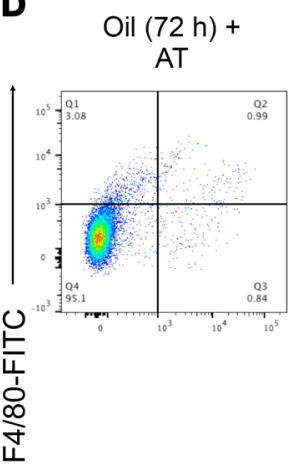

$\mathrm{CCl}_{4}(72 \mathrm{~h})$ injury +
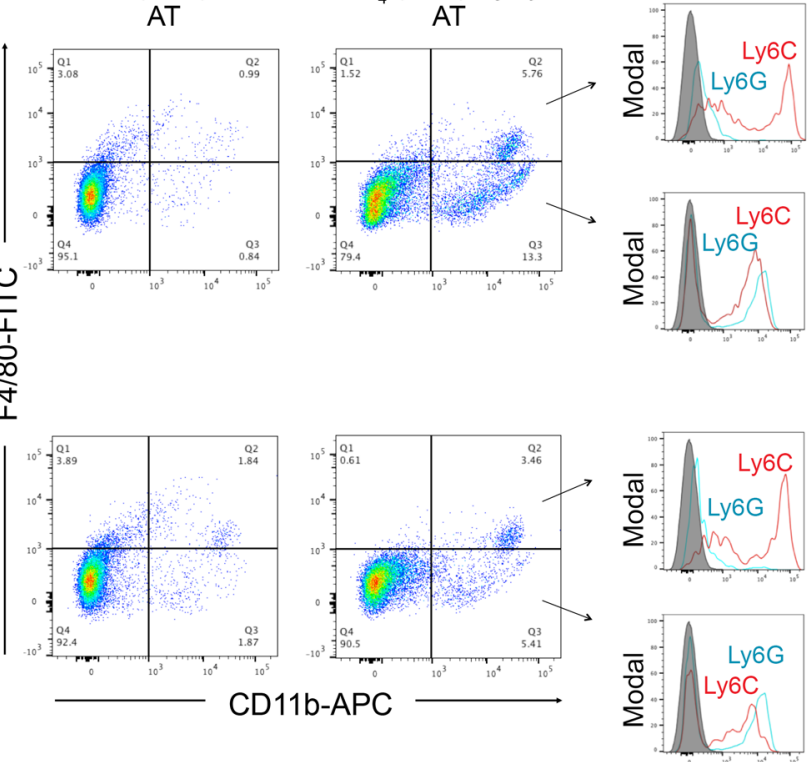

G

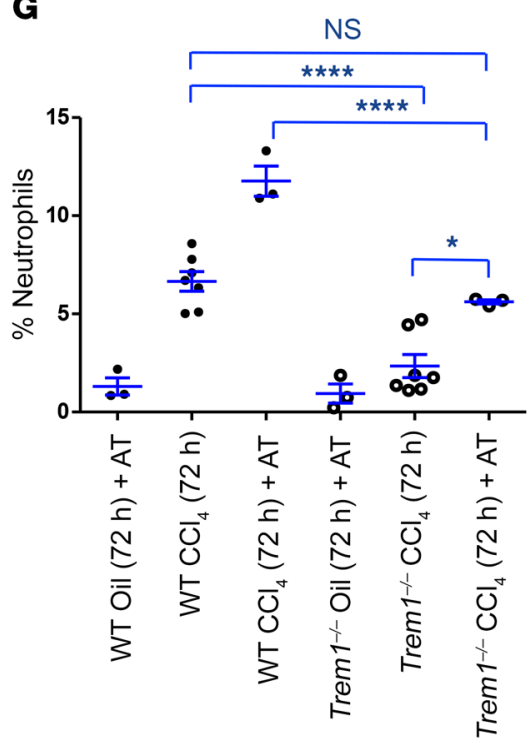

H

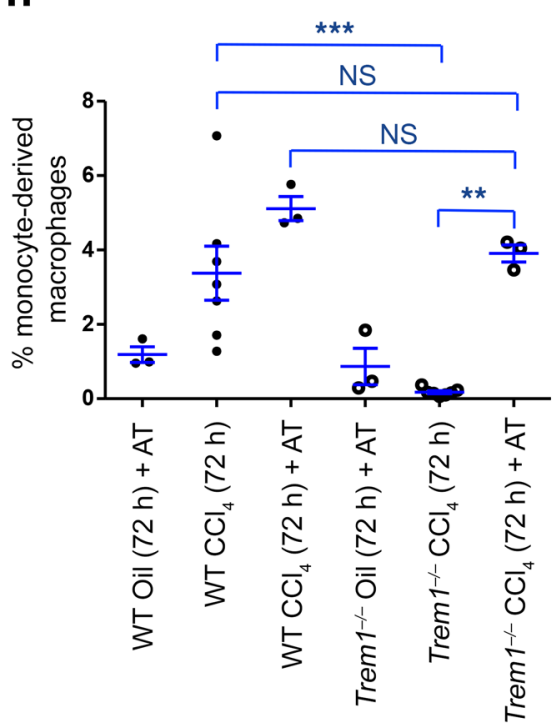

B

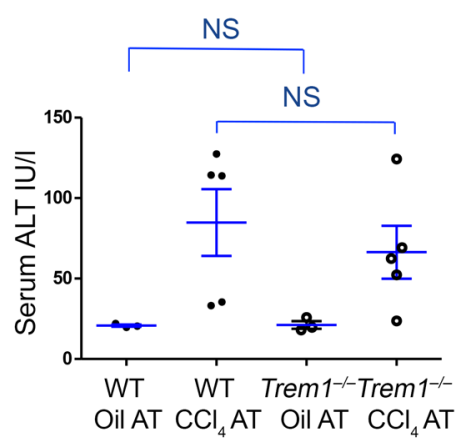

C

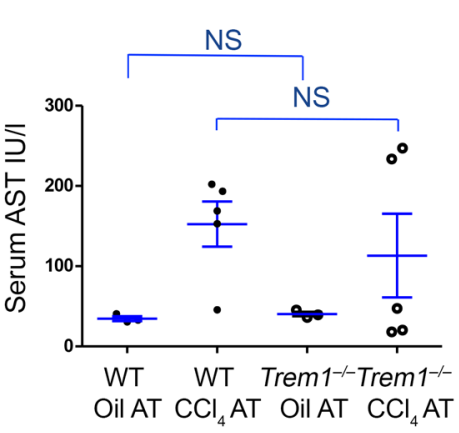

E

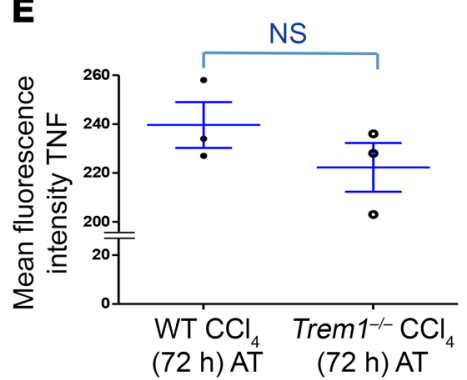

F

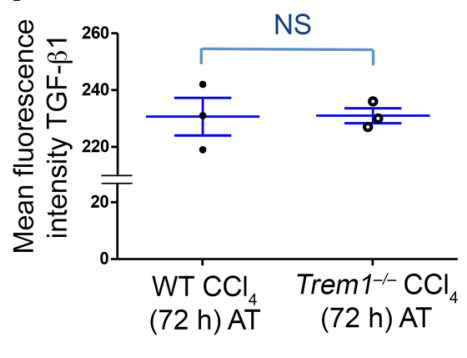


Figure 7. Adoptive transfer of Kupffer cells from WT mice increases liver injury and recruitment of inflammatory cells in Trem 1/-- mice. (A) Representative images of FITC-conjugated anti-F4/80 antibody-stained liver sections from the indicated mice 72 hours after injection of clodronate-containing liposomes ( $n=3$ mice/group, top), followed by reconstitution of predepleted livers with WT Kupffer cells ( $n=3$ mice/group). Adoptively transferred cells were observed at 72 hours by immunofluorescence staining. Original magnification, $\times 40$; scale bars: $20 \mu \mathrm{m}$. Images shown are representative of 2 independent experiments. Levels of (B) ALT and (C) AST in serum from WT and Trem 1/-- mice with adoptive transfer of WT Kupffer cells followed by a single dose of $\mathrm{CCl}_{4}$ treatment were measured 72 hours after $\mathrm{CCl}_{4}$ injury by colorimetric assay ( $n=3-5$ mice/group). (D) Macrophage-depleted WT and Trem1/-- mice were adoptively transferred with WT Kupffer cells following treatment with a single dose of $\mathrm{CCl}_{4}$. Flow cytometric dot plots of liver cells stained with anti-F4/80, anti-CD11b, anti-TNF, anti-TGF- $\beta 1$, anti-Ly6C, anti-Ly6C, anti-CCR2, and anti-CX3CR1 antibodies ( $n=3$ mice/group). Flow cytometric histograms of Ly6C, Ly6G, CCR2, and CX3CR1 expression shown on gated F4/80+CD11 $\mathrm{b}^{+}$and $\mathrm{F} 4 / 80^{-} \mathrm{CD}_{11} \mathrm{~b}^{+}$cells ( $n=3$ mice/group). Control staining was performed with IgG isotype (gray histograms). Mean fluorescence intensity of TNF (E) and TCF- $\beta 1$ (F) in the indicated mice is shown for gated F4/80 ${ }^{+}$CD11 $b^{-}$Kupffer cells ( $n=3$ mice/group). (G and $\mathbf{H}$ ) Percentage of liver-infiltrated neutrophils and inflammatory monocytes under the indicated experimental conditions in WT and Trem1-/- mice ( $n=3-7 /$ group). Results are displayed as the mean \pm SEM. ${ }^{* *} P<0.01,{ }^{* *} P<0.001$, and ${ }^{* * *} P<0.0001$, by 2 -tailed Student's $t$ test (B, C, E, and $\mathbf{F}$ ) and ANOVA followed by Bonferroni's post hoc test ( $\mathbf{G}$ and $\mathbf{H})$. AT, adoptive transfer.

ferred Kupffer cells migrated at 18 hours into the livers of depleted $\mathrm{WT}$ and Trem1 $^{-/-}$recipient mice. Trem1 $1^{-/}$mice in which Trem1sufficient Kupffer cells were transferred recovered the ability to increase serum ALT and AST enzymes 72 hours after $\mathrm{CCl}_{4}$ injection at comparable levels, as observed in WT mice (Figure 4, C and F, and Figure 7, B and C). Adoptively transferred Trem1-sufficient Kupffer cells produced comparable amounts of TNF and TGF- $\beta 1$ in WT and Trem1 ${ }^{-1-}$ recipient mice following $\mathrm{CCl}_{4}$ treatment (Figure 7, D-F). The recruitment of peripheral blood neutrophils after $\mathrm{CCl}_{4}$ treatment was comparable between Trem1 $^{-/}$and WT mice reconstituted with Trem1-sufficient Kupffer cells (Supplemental Figure 10, A and B). Also, $\mathrm{CCl}_{4}$ treatment of Trem1 ${ }^{-/-}$mice reconstituted with Trem1-sufficient Kupffer cells induced a similar increase in the number of $\mathrm{CD}_{11} \mathrm{~b}^{+} \mathrm{F} 4 / 80^{+}$patrolling monocytes/ macrophages at 72 hours, as observed in $\mathrm{CCl}_{4}$-injured WT mice (Supplemental Figure 10, A and C). The numbers of inflammatory monocytes and neutrophils recruited 72 hours after $\mathrm{CCl}_{4}$ treatment to the livers of Trem1 $1^{-/}$mice reconstituted with Trem1sufficient Kupffer cells were comparable to those observed in WT mice, although the numbers were lower than in WT mice reconstituted with Trem1-sufficient Kupffer cells (Figure 7, D, G, and H). Conversely, adoptive transfer of Trem1-deficient Kupffer cells into WT mice resulted in reduced $\mathrm{CCl}_{4}$-induced liver injury and altered recruitment of neutrophils as well as of patrolling and inflammatory monocytes/macrophages (Supplemental Figure 11, A-H). These data indicate that transferred Trem1-sufficient Kupffer cells were activated in Trem1 $1^{-1-}$ mice in response to $\mathrm{CCl}_{4}$ exposure and could induce liver injury, while the transfer of Trem1-deficient Kupffer cells into WT mice protected them from $\mathrm{CCl}_{4}$-induced liver injury.

Increased liver infiltration with TREM-1-positive cells, including Kupffer cells and monocytes/macrophages, in patients with hepatic fibrosis. Human liver tissues from a control group $(n=6)$ and from patients with liver fibrosis $(n=5)$ were analyzed for fibrosis biomarkers, TREM-1 expression, and phenotype. We found that collagen deposition, evaluated by Masson's trichrome staining, and $\alpha$-SMA expression levels were significantly increased in patients with advanced liver fibrosis (Figure $8, \mathrm{~A}-\mathrm{C}$, left panels, and D, and Supplemental Figures 12 and 13). In normal liver, most TREM-1positive cells were found in and around the hepatic sinusoid, which is the primary location of Kupffer cells and of the morphologically and functionally unique LSECs (Figure 8C, middle and right panels). Since LSECs play a major role in liver regeneration after liver injury, it will be interesting to determine the role of TREM-1 in LSEC functions, especially in their crosstalk with HSCs during fibrogenesis. Healthy (noncapillarized) LSECs prevent and reverse the activation of HSCs $(35,36)$. However, during fibrosis, capillarized LSECs lose the ability to antagonize HSC activation (36). We detected a similar number of TREM-1-positive cells in normal liver tissues from the control group and in nonfibrotic areas from patients with advanced liver fibrosis $(31.47 \% \pm 13.47 \%$ and $27.25 \%$ $\pm 6.8 \%$, respectively) (Figure $8 \mathrm{C}$, middle panels). In contrast, we observed an increase in the number of TREM-1-positive cells in fibrotic areas compared with nonfibrotic areas $(82.04 \% \pm 9.68 \%$, Figure 8, C, middle panels, and E, and Supplemental Figure 14). TREM-1-positive cells that express CD68, a marker of Kupffer cells, were significantly increased in fibrotic areas (Figure 8, F and $\mathrm{H}$, and Supplemental Figure 15). Other TREM-1-positive cells that were also substantially increased in fibrotic areas expressed the CD11b marker and were most markedly increased in these fibrotic areas; the majority of these cells were myeloid cells, including monocytes and monocyte-derived macrophages (Figure 8, F and G, and Supplemental Figure 16). Together, these data suggest that human liver fibrosis is associated with the recruitment and differentiation of TREM-1-positive Kupffer cells and monocytes and monocyte-derived macrophages.

\section{Discussion}

Hepatic fibrosis is the consequence of liver damage produced by chronic infectious hepatitis, alcohol, or chemical insults (1-4). An excessive inflammatory response to the initial liver damage mediated by viruses or toxic metabolites amplifies the tissue damage, resulting in progression to chronic liver injury and fibrosis that eventually lead to cirrhosis and organ failure. Since fibrosis is preceded by an inflammatory response, targeting of both the innate and adaptive immune systems may provide powerful tools for therapeutic approaches to prevent, slow, or even reverse fibrosis or cirrhosis $(37,38)$.

The TREM-1 signaling pathway has emerged as a central component of the liver's inflammatory response during acute and chronic liver injury. In this study, we have shown that the TREM-1-mediated response to the initial liver damage causes further injury to the liver and is linked to the development of fibrosis. We focused in detail on the innate immune response to $\mathrm{CCl}_{4}$-induced tissue damage and found that the activation of TREM-1 in Kupffer cells plays a key role in initiation of the liver's inflammatory response followed by HSC activation during fibrogenesis (Supplemental Figure 17). Kupffer cells are the major population of cells that express TREM-1 in the liver. During $\mathrm{CCl}_{4}$-induced fibrogenesis, the expression of TREM-1 on 
A
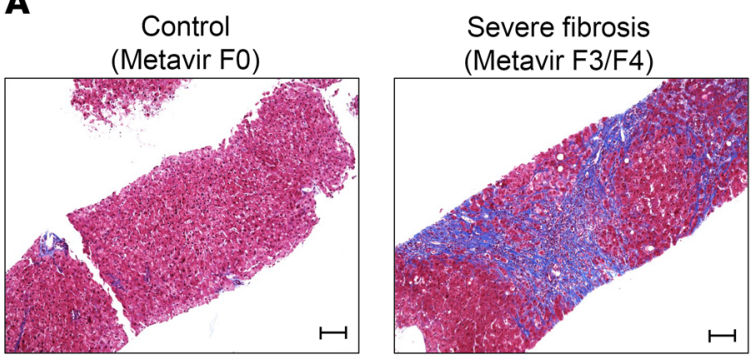

c Metavir FO)

Severe Fibrosis (Metavir F3/F4)
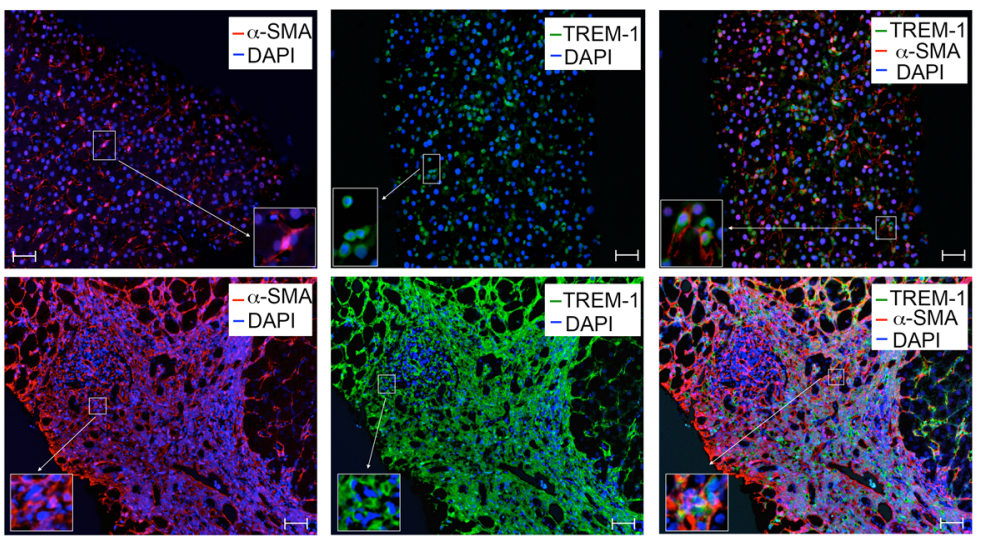

$\mathbf{F}$
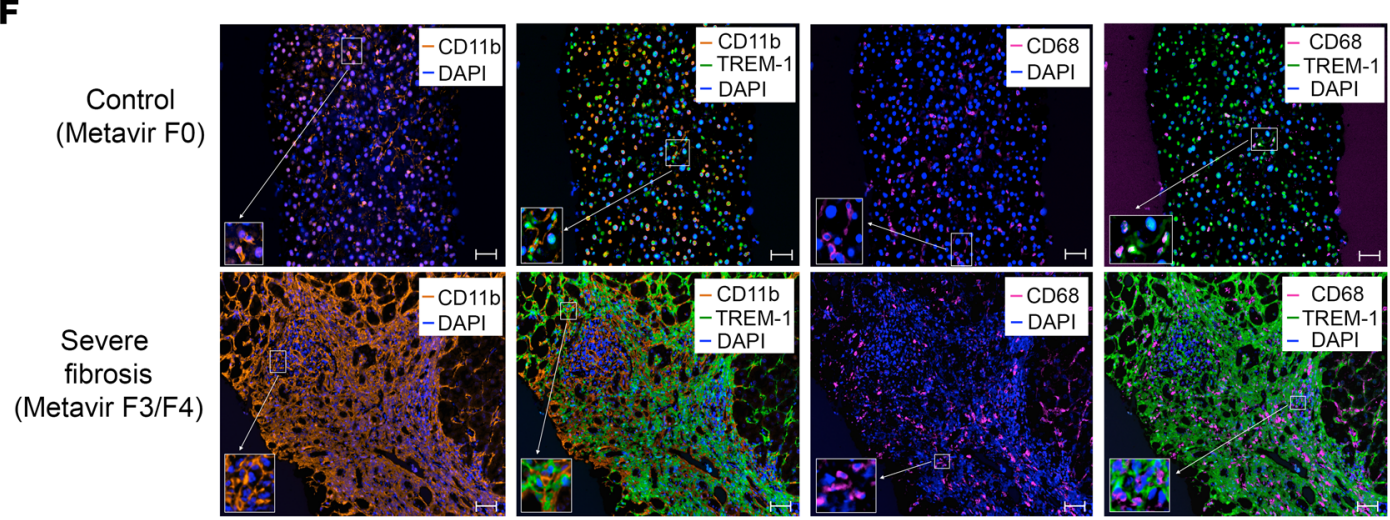

B
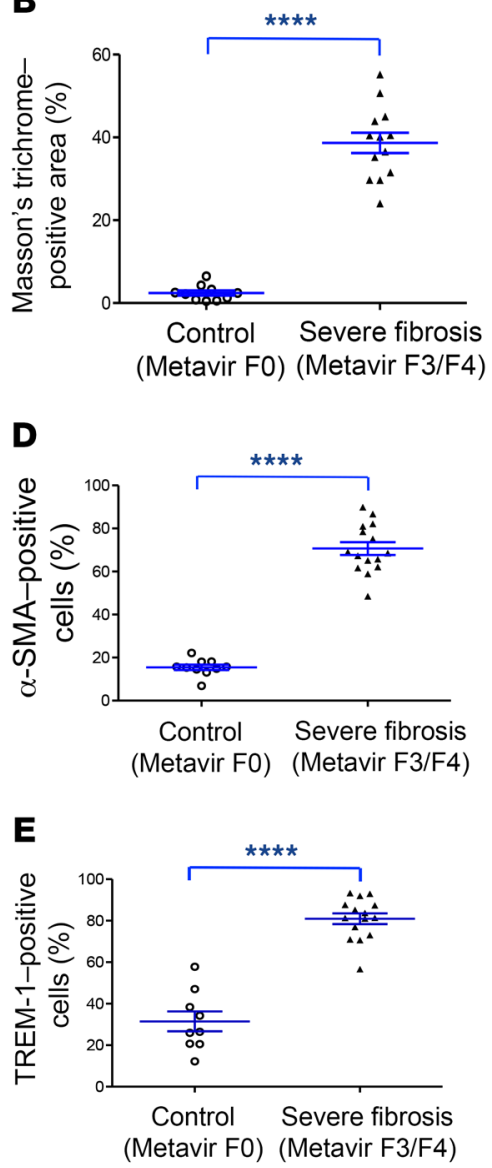

G

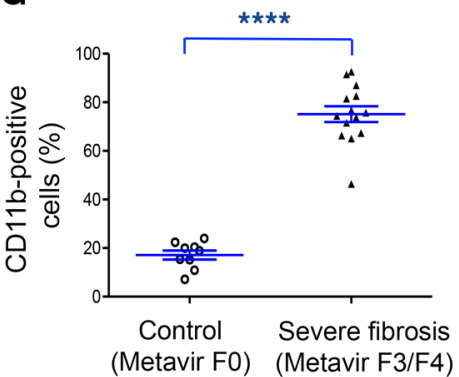

H

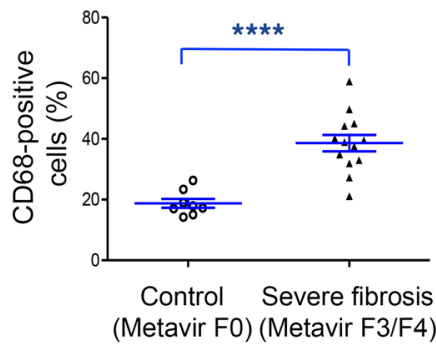


Figure 8. Increased TREM-1-, CD11b-, and CD68-positive cell infiltration in human liver samples from patients diagnosed with advanced fibrosis.

(A) Representative images of human liver samples from a control subject (Metavir = FO, $n=6$ ) and a patient diagnosed with advanced fibrosis (Metavir = F3/F4, $n=4$ ). Samples were stained with Masson's trichrome to assess collagen deposition. Original magnification, $\times 10$; scale bars: $100 \mu \mathrm{m}$. (B) Quantification (percentage) of Masson's trichrome-positive areas in liver samples from a control subject ( $n=10$ areas) and a patient with advanced fibrosis ( $n=13$ areas). (C) Fluorescence multiplexed IHC of human liver samples. Samples from a control subject and a patient with advanced fibrosis were stained for $\alpha$-SMA (red), TREM-1 (green), and DAP (blue). Original magnification, $\times 20$; scale bars: $50 \mu \mathrm{m}$. (D) Quantification of $\alpha$-SMA-positive cells (percentage) from a control subject and a patient with advanced fibrosis ( $n=10$ areas vs. $n=15$ areas, respectively. (E) Quantification (percentage) of TREM-1-positive cells from a control subject and a patient with advanced fibrosis ( $n=9$ and $n=14$ areas, respectively). (F) Samples from a control subject and a patient with advanced fibrosis were stained for CD11b (orange), CD68 (magenta), TREM-1 (green), and DAPI (blue). Original magnification, $\times 20$; scale bars: $50 \mu \mathrm{m}$. (G) Quantification (percentage) of CD11b-positive cells from a control subject and a patient with advanced fibrosis ( $n=10$ vs. $n=15$ areas, respectively). (H) Quantification (percentage) of CD68-positive cells from a control subject and a patient with advanced fibrosis ( $n=8$ and $n=12$ areas, respectively). Data are displayed as the mean \pm SEM. ${ }^{* * *} P<0.0001$, by 2 -tailed Student's $t$ test (B, D, E, G, and $\mathbf{H})$.

Kupffer cells was enhanced early and maintained at levels higher than those in untreated mice for the duration of the experiment. TREM-1 activation enhanced the inflammatory response to tissue damage, amplifying liver damage and leading to chronic inflammation. Recognition of HMGB1 by TREM-1 on Kupffer cells leads to the production of proinflammatory cytokines (IL-1 $\beta$, TNF, IL-6) that sensitize hepatocytes to apoptosis and necrosis $(21,29,31,32)$. Our study defines several crucial components of the TREM-1-mediated inflammatory response in the liver. The number of TREM-1-activated Kupffer cells and monocytederived macrophages was markedly increased in fibrotic livers. The extensive recruitment of cells to the injured liver and/ or survival and proliferation of resident cells could explain this finding $(39,40)$. There are conflicting results on the role of TREM-1 in prolonging the lifespan of myeloid cells. KlesneyTait et al. (41) demonstrated that TREM-1- and TREM-3deficient neutrophils undergo cell death at a rate similar to that of WT neutrophils, but these cells failed to migrate into the intrapulmonary airspace in a murine pneumonia model. In contrast, in various other models, TREM-1 blockade or deficiency was observed to inhibit cell proliferation directly or indirectly (21), suggesting that a full understanding of the role of TREM-1 in cell survival requires further investigation. Our study defines the recruitment of proinflammatory cells to the injured liver as one of the major mechanisms by which TREM-1 signaling on activated Kupffer cells establishes a chronic inflammatory response and enhances liver damage. Trem1deficient Kupffer cells in $\mathrm{CCl}_{4}$-treated mice fail to express genes such as $\mathrm{Ccl} 9, \mathrm{Cxcl} 2$, and $\mathrm{Cxcl} 3$ that encode chemokines involved in the recruitment, differentiation, and function of monocytes, neutrophils and macrophages (39, 40, 42-44). Our findings demonstrated that a high number of infiltrating proinflammatory monocytes and monocyte-derived macrophages were recruited from bone marrow and peripheral blood to the area of injured prefibrotic and fibrotic livers in TREM-1-positive mice. Remarkably, we observed absent or reduced numbers of infiltrating monocytes and monocyte-derived macrophages in Trem1-deficient mice.

In our study, HSCs in normal, healthy livers did not express TREM-1. There is increasing evidence that inflammationrelated malignancy may trigger the expression of TREM-1 on tumor-activated HSCs (20). We did not detect Trem 1 expression on HSCs isolated from control (oil-treated) mice or during early stages of fibrogenesis in WT mice. However, we detected an upregulation of Trem $1 \mathrm{mRNA}$ on activated HSCs in $50 \%$ of the WT mice treated with $\mathrm{CCl}_{4}$ for 6 weeks (data not shown). Since activation of HSCs occurs rapidly after liver injury, TREM-1 signaling is not the primary target on HSCs that drives fibrogenesis. Our study provides several lines of evidence that TREM-1 signaling on Kupffer cells is the main target that drives fibrogenesis. Activated Kupffer cells from Trem-1+/+ mice release various proinflammatory mediators, including the chemokine CCL9 as well as TGF- $\beta 1$, which is considered the most powerful mediator of HSC activation $(1,13)$. Kupffer cells are the main source of TGF- $\beta 1$ in the liver and promote HSC activation and fibrogenesis $(13,45)$. Several signaling pathways modulate hepatic inflammation and fibrogenesis. In particular, TLR4 enhances hepatic fibrosis through TGF- $\beta 1$ signaling. The expression of TLR4 has been demonstrated on many cells in the liver, including Kupffer cells and HSCs. Tlr4-deficent mice demonstrated markedly decreased fibrogenesis (13). However, chimeric mice containing Tlr4-deficient Kupffer cells had normal fibrogenesis (13). These data suggest that in Kupffer cells, TREM-1 rather than TLR4 plays a major role in promoting fibrogenesis. Moreover, adoptive transfer of Trem1-sufficient Kupffer cells into either $\mathrm{Trem1}^{-/}$or WT mice resulted in similar $\mathrm{CCl}_{4}$ induced liver injury. Trem1-sufficient Kupffer cells were activated and produced comparable amounts of TNF and TGF- $\beta 1$ in both WT and $\mathrm{CCl}_{4}$-treated Trem1 $1^{-/-}$mice. Strikingly, Trem1-sufficient Kupffer cells could recruit patrolling monocytes into the peripheral blood and inflammatory infiltrating monocytes into the livers of Trem1 ${ }^{-1-}$ mice. Recently, new players in TGF- $\beta 1$-mediated liver fibrogenesis have been discovered, one of which is the Notch signaling pathway $(46,47)$. Notch pathway components were found to be significantly upregulated in TGF- $\beta 1$-activated HSCs, inflammatory monocytes, macrophages, and in mouse and human fibrotic livers (47). Our data demonstrated that TREM-1positive Kupffer cells significantly upregulated the expression of a Notch ligand (Jag1) at an early stage of liver injury, supporting the idea that Notch signaling in Kupffer cells may drive liver fibrosis by promoting crosstalk between Kupffer cells and HSCs. Although TGF- $\beta 1$ is the major regulator of fibrogenesis, TGF- $\beta 1-$ independent pathways may also be involved. These new pathways include the emerging role of oncostatin M (OSM), an inflammatory cytokine of the IL- 6 family that has been discovered in macrophage activation and lung fibrogenesis (48). Our data indicate a link between the expression of Osm and TREM-1 signaling in Kupffer cells, which supports the concept that OSM from Kupffer cells may contribute to liver fibrogenesis.

Our data demonstrate that the TREM-1 pathway on Kupffer cells plays an essential role in hepatic inflammation and fibrogenesis in a mouse model of fibrosis. Furthermore, analysis of human 
liver tissue samples demonstrated robustly increased infiltration of TREM-1-positive Kupffer cells and monocytes/macrophages in fibrotic areas in patients with liver fibrosis. Therefore, interference with the functions of TREM-1 on Kupffer cells could provide a promising therapeutic approach, enabling the treatment of an overwhelming inflammatory liver response and fibrotic liver disease. Because several fibrogenic pathways are conserved across tissues, our findings on the role of TREM-1 in liver tissue-resident macrophages could be expanded to the study of fibrosis in the lungs, heart, kidneys, and other organs.

\section{Methods}

Animals and fibrosis induction. Trem1-deficient mice were generated in our laboratory. Liver fibrosis was induced by $\mathrm{CCl}_{4}$ peritoneal injection. See the Supplemental Methods for details.

Measurement of hepatic collagen deposition and cell infiltration. Collagen deposition was measured with a Picrosirius Red Stain Kit or Masson's trichrome staining (both from IHC World), and cell infiltration was measured by H\&E staining. Further details are provided in the Supplemental Methods.

Immunofluorescence, IHC, staining with the Opal Multiplex method and tyramide signal amplification plus fluorescent reagents, RT-qPCR, and immunoblotting. These assays were performed using standard procedures. For detailed information, see the Supplemental Methods.

ALT and AST. Blood from WT and Trem1-1- mice treated with oil (control) or $\mathrm{CCl}_{4}$ was collected, and sera were extracted from the blood. Serum ALT and AST levels were measured with the colorimetric assays Liquid ALT (SGPT) and Liquid AST (SGOT) (Pointe Scientific) according to the manufacturer's recommendations.

Isolation of bone marrow cells, liver nonparenchymal cells, and Kupffer cells from mice. Bone marrow cells were isolated from mouse femurs. Liver nonparenchymal cells and Kupffer cells were isolated by collagenase digestion in vivo, followed by further collagenase digestion in vitro. Cells were isolated by density gradient centrifugation, followed by MACS magnetic bead separation. See the Supplemental Methods for details.

Isolation of HSCs. HSCs were isolated by in vivo digestion with a mixture of collagenase and pronase and further digested in vitro, followed by density gradient centrifugation. See the Supplemental Methods for further information.

Antibodies and flow cytometric analysis. Cells from bone marrow and blood and nonparenchymal cells from mouse livers were isolated and labeled with different antibodies. For more information concerning antibodies and staining conditions, see the Supplemental Methods.

RNA-seq and data analysis. Total RNA from Kupffer cells was extracted using TRIzol (Thermo Fisher Scientific), and RNA-seq and analysis was performed. Additional information is provide in the Supplemental Methods. RNA-seq data were deposited in the NCBI's Gene Expression Omnibus (GEO) database (GEO GSE116236).

Depletion and adoptive transfer of Kupffer cells. Depletion of Kupffer cells in Trem1-deficient mice was performed with clodronatecontaining liposome suspension. Kupffer cells from WT mice were isolated and adoptively transferred into recipient Trem1-deficient mice. For detailed information, see the Supplemental Methods.

Quantitative assessment of the uptake and distribution of reticuloendothelial system-specific nanoparticles during liver fibrogenesis. An assay to determine the uptake and distribution of reticuloendothelial system-specific nanoparticles during liver fibrogenesis was performed with Mvivo BIS nanoparticles and a small animal CT scanner. Further details are available in the Supplemental Methods.

Human tissue. Deidentified, formalin-fixed, paraffin-embedded liver tissue specimens from healthy controls $(n=6)$ and patients with liver fibrosis $(n=5)$ were obtained through US Biomax Inc. and the Georgia Cancer Center Biorepository. Liver fibrosis was evaluated according to Metavir staging. Significant fibrosis was defined as Metavir stages $\mathrm{F} \geq 2$, severe fibrosis as Metavir $\mathrm{F} \geq 3$, and cirrhosis as F4 (49). Masson's trichrome and fluorescence multiplexed IHC staining of human liver specimens and analysis are described in detail in the Supplemental Methods.

Statistics. All data represent the mean \pm SEM. Comparisons of 2 groups were analyzed using an unpaired, 2-tailed Student $t$ test. Comparisons of 3 or more groups were analyzed using ANOVA with a Bonferroni's test when multiple pairwise comparisons were made between different groups. For all analyses, a $P$ value of less than 0.05 was considered significant. Analyses were performed using GraphPad Prism, version 5.04. For human liver samples, statistical analysis was performed using SAS 9.4 statistical software (SAS Institute Inc.). A 1-way ANOVA with Bonferroni's correction was used for multiple pairwise comparisons between 3 human-related groups, after adjusting for multiplicity. As an exploratory analysis, least squares mean testing was used to test the difference between any pair of subjects. A $P$ value of less than 0.05 was considered statistically significant.

Study approval. All mice were maintained under specific pathogenfree conditions at Augusta University with IACUC approval (protocol 2008-0051). Animal studies were performed in strict accordance with the recommendations in the NIH Guide for the Care and Use of Laboratory Animals (National Academies Press, 2011). Analysis of human formalin-fixed, paraffin-embedded tissue specimens was approved by the Augusta University IRB (protocol 1023370).

\section{Author contributions}

$\mathrm{AA}, \mathrm{VPD}, \mathrm{DDH}$, and $\mathrm{AD}$ designed and conducted experiments and analyzed results. ASA provided support for image acquisition and analysis. RS analyzed results. ATNL, GT, and AH designed and conducted experiments, analyzed results, and wrote the manuscript.

\section{Acknowledgments}

This work was supported by NIH grant CA172230 (to AH). We thank R. B. Markowitz (Georgia Cancer Center, Augusta University) for critical reading of the manuscript. We thank Houssein Abdul Sater, Roni Bollag, and Kimya Jones of the Georgia Cancer Center Biorepository for help with obtaining liver samples and for excellent technical support. We also thank Maciej Zerkowski (Perkin Elmer, New York, New York, USA) for technical advice in analyzing multispectral images and cell phenotypes. We are grateful to the Georgia Cancer Center community for fruitful discussions and support.

Address correspondence to: Anatolij Horuzsko, Molecular Oncology and Biomarkers Program, Georgia Cancer Center, Department of Medicine, Medical College of Georgia, Augusta University, 1410 Laney Walker Boulevard, Augusta, Georgia, 30912, USA. Phone: 706.721.8736; Email: ahoruzsko@augusta.edu. 
1. Bataller R, Brenner DA. Liver fibrosis. J Clin Invest. 2005;115(2):209-218.

2. Hernandez-Gea V, Friedman SL. Pathogenesis of liver fibrosis. Annu Rev Pathol. 2011;6:425-456.

3. Bataller R, Gao B. Liver fibrosis in alcoholic liver disease. Semin Liver Dis. 2015;35(2):146-156.

4. Schwabe R, Bataller R. Liver fibrosis. Foreword. Semin Liver Dis. 2015;35(2):95-96.

5. Rosselli M, Lotersztajn S, Vizzutti F, Arena U, Pinzani M, Marra F. The metabolic syndrome and chronic liver disease. Curr Pharm Des. 2014;20(31):5010-5024.

6. Koyama Y, Brenner DA. Liver inflammation and fibrosis. J Clin Invest. 2017;127(1):55-64.

7. Akira S, Takeda K. Toll-like receptor signalling. Nat Rev Immunol. 2004;4(7):499-511.

8. Adams DH, Ju C, Ramaiah SK, Uetrecht J, Jaeschke H. Mechanisms of immune-mediated liver injury. Toxicol Sci. 2010;115(2):307-321.

9. Woolbright BL, Jaeschke H. The Impact of Sterile Inflammation in Acute Liver Injury. J Clin Transl Res. 2017;3(Suppl 1):170-188.

10. Tomasek JJ, Gabbiani G, Hinz B, Chaponnier C, Brown RA. Myofibroblasts and mechanoregulation of connective tissue remodelling. Nat Rev Mol Cell Biol. 2002;3(5):349-363.

11. Kubes P, Mehal WZ. Sterile inflammation in the liver. Gastroenterology. 2012;143(5):1158-1172.

12. Woolbright BL, Jaeschke H. Sterile inflammation in acute liver injury: myth or mystery? Expert Rev Gastroenterol Hepatol. 2015;9(8):1027-1029.

13. Seki E, et al. TLR4 enhances TGF-beta signaling and hepatic fibrosis. Nat Med. 2007;13(11):1324-1332.

14. Wynn TA, Ramalingam TR. Mechanisms of fibrosis: therapeutic translation for fibrotic disease. Nat Med.2012;18(7):1028-1040.

15. Ford JW, McVicar DW. TREM and TREM-like receptors in inflammation and disease. Curr Opin Immunol. 2009;21(1):38-46.

16. Bouchon A, Dietrich J, Colonna M. Cutting edge: inflammatory responses can be triggered by TREM-1, a novel receptor expressed on neutrophils and monocytes. JImmunol. 2000;164(10):4991-4995.

17. Bouchon A, Facchetti F, Weigand MA, Colonna M. TREM-1 amplifies inflammation and is a crucial mediator of septic shock. Nature. 2001;410(6832):1103-1107.

18. Lanier LL. Natural killer cell receptor signaling. Curr Opin Immunol. 2003;15(3):308-314.

19. Turnbull IR, Colonna M. Activating and inhibitory functions of DAP12. Nat Rev Immunol. 2007;7(2):155-161.

20. Liao R, et al. Expression of TREM-1 in hepatic stellate cells and prognostic value in hepatitis
B-related hepatocellular carcinoma. Cancer Sci. 2012;103(6):984-992.

21. Wu J, Li J, Salcedo R, Mivechi NF, Trinchieri G, Horuzsko A. The proinflammatory myeloid cell receptor TREM-1 controls Kupffer cell activation and development of hepatocellular carcinoma. Cancer Res. 2012;72(16):3977-3986.

22. Pierce RA, Glaug MR, Greco RS, Mackenzie JW, Boyd CD, Deak SB. Increased procollagen mRNA levels in carbon tetrachloride-induced liver fibrosis in rats. J Biol Chem. 1987;262(4):1652-1658.

23. Oumi N, Taniguchi KA, Kanai AM, Yasunaga M, Nakanishi T, Sato K. A crucial role of bone morphogenetic protein signaling in the wound healing response in acute liver injury induced by carbon tetrachloride. Int J Hepatol. 2012;2012:476820.

24. Friedman SL. Hepatic fibrosis -- overview. Toxicology. 2008;254(3):120-129.

25. Mann DA, Marra F. Fibrogenic signalling in hepatic stellate cells. J Hepatol. 2010;52(6):949-950.

26. Mederacke I, Dapito DH, Affò S, Uchinami H, Schwabe RF. High-yield and high-purity isolation of hepatic stellate cells from normal and fibrotic mouse livers. Nat Protoc. 2015;10(2):305-315.

27. Mederacke I, et al. Fate tracing reveals hepatic stellate cells as dominant contributors to liver fibrosis independent of its aetiology. Nat Commun. 2013;4:2823.

28. McCay PB, Lai EK, Poyer JL, DuBose CM, Janzen EG. Oxygen- and carbon-centered free radical formation during carbon tetrachloride metabolism. Observation of lipid radicals in vivo and in vitro. J Biol Chem . 1984;259(4):2135-2143.

29. El Mezayen R, El Gazzar M, Seeds MC, McCall CE, Dreskin SC, Nicolls MR. Endogenous signals released from necrotic cells augment inflammatory responses to bacterial endotoxin. Immunol Lett. 2007;111(1):36-44.

30. Brenner C, Galluzzi L, Kepp O, Kroemer G. Decoding cell death signals in liver inflammation. J Hepatol. 2013;59(3):583-594.

31. Thankam FG, Dilisio MF, Dietz NE, Agrawal DK. TREM-1, HMGB1 and RAGE in the Shoulder Tendon: Dual Mechanisms for Inflammation Based on the Coincidence of Glenohumeral Arthritis. PLoS ONE. 2016;11(10):e0165492.

32. Subramanian S, Pallati PK, Sharma P, Agrawal DK, Nandipati KC. Significant association of TREM-1 with HMGB1, TLRs and RAGE in the pathogenesis of insulin resistance in obese diabetic populations. Am J Transl Res. 2017;9(7):3224-3244.

33. Li L, et al. The chemokine receptors CCR2 and CX3CR1 mediate monocyte/macrophage trafficking in kidney ischemia-reperfusion injury.
Kidney Int. 2008;74(12):1526-1537.

34. Tacke F, et al. Monocyte subsets differentially employ CCR2, CCR5, and CX3CR1 to accumulate within atherosclerotic plaques. JClin Invest. 2007;117(1):185-194.

35. Deleve LD, Wang X, Guo Y. Sinusoidal endothelial cells prevent rat stellate cell activation and promote reversion to quiescence. Hepatology. 2008;48(3):920-930.

36. DeLeve LD. Liver sinusoidal endothelial cells and liver regeneration. J Clin Invest. 2013;123(5):1861-1866.

37. Trautwein C, Friedman SL, Schuppan D, Pinzani M. Hepatic fibrosis: Concept to treatment. J Hepatol. 2015;62(1 Suppl):S15-S24.

38. Fallowfield JA. Future mechanistic strategies for tackling fibrosis--an unmet need in liver disease. Clin Med (Lond). 2015;15 Suppl 6:s83-s87.

39. Marra F, Aleffi S, Galastri S, Provenzano A Mononuclear cells in liver fibrosis. Semin Immunopathol. 2009;31(3):345-358.

40. Marra F, Tacke F. Roles for chemokines in liver disease. Gastroenterology. 2014;147(3):577-594.e1.

41. Klesney-Tait J, et al. Transepithelial migration of neutrophils into the lung requires TREM-1. J Clin Invest. 2013;123(1):138-149.

42. Kolaczkowska E, Kubes P. Neutrophil recruitment and function in health and inflammation. Nat Rev Immunol. 2013;13(3):159-175.

43. Stock AT, Smith JM, Carbone FR. Type I IFN suppresses Cxcr2 driven neutrophil recruitment into the sensory ganglia during viral infection. JExp Med. 2014;211(5):751-759.

44. Rydström A, Wick MJ. Monocyte and neutrophil recruitment during oral Salmonella infection is driven by MyD88-derived chemokines. Eur J Immunol. 2009;39(11):3019-3030.

45. Koyama Y, et al. Mesothelin/mucin 16 signaling in activated portal fibroblasts regulates cholestatic liver fibrosis. J Clin Invest. 2017;127(4):1254-1270.

46. Geisler F, Strazzabosco M. Emerging roles of Notch signaling in liver disease. Hepatology. 2015;61(1):382-392.

47. Bansal R, van Baarlen J, Storm G, Prakash J. The interplay of the Notch signaling in hepatic stellate cells and macrophages determines the fate of liver fibrogenesis. Sci Rep. 2015;5:18272.

48. Wong S, Botelho FM, Rodrigues RM, Richards CD. Oncostatin M overexpression induces matrix deposition, STAT3 activation, and SMAD1 Dysregulation in lungs of fibrosis-resistant BALB/c mice. Lab Invest. 2014;94(9):1003-1016.

49. Boursier J, et al. A new combination of blood test and fibroscan for accurate non-invasive diagnosis of liver fibrosis stages in chronic hepatitis C. Am J Gastroenterol. 2011;106(7):1255-1263. 